\title{
A Class of Stochastic Programs with Decision Dependent Uncertainty
}

\author{
Vikas Goel*and Ignacio E. Grossmann ${ }^{\dagger}$ \\ Department of Chemical Engineering, Carnegie Mellon University, \\ 5000 Forbes Avenue, Pittsburgh, Pennsylvania 15213
}

\begin{abstract}
The standard approach to formulating stochastic programs is based on the assumption that the stochastic process is independent of the optimization decisions. We address a class of problems where the optimization decisions influence the time of information discovery for a subset of the uncertain parameters. We extend the standard modeling approach by presenting a disjunctive programming formulation that accommodates stochastic programs for this class of problems. A set of theoretical properties that lead to reduction in the size of the model is identified. A Lagrangean duality based branch and bound algorithm is also presented.
\end{abstract}

\section{Introduction}

Stochastic programming deals with the problem of making optimal decisions in the presence of uncertainty. In stochastic programs, the uncertainty is represented by probability distributions and the interaction between the stochastic and decisions processes is modeled so that the decisionmaker has the option of adjusting the decisions based on how the uncertainty unfolds. From the modeling perspective, most previous work in the stochastic programming literature deals with problems with exogenous uncertainty (Jonsbraten (1998)), where the optimization decisions cannot influence the stochastic process.

Pflug (1990) was the first to address the case with endogenous uncertainty, where the underlying stochastic process depends on the optimization decisions. Previous work on this class of uncertainty is limited to a few papers only. Since this paper deals with endogenous uncertainty, we only review

\footnotetext{
*E-mail: vgoel@andrew.cmu.edu

${ }^{\dagger}$ To whom all correspondence should be addressed. Tel.: (412) 268-3642. Fax: (412) 268-7139. E-mail: grossmann@cmu.edu.
} 
the previous work in the stochastic programming literature on this type of uncertainty. To motivate the need for this paper, we also present brief descriptions of some real world problems with this type of uncertainty. Reviews of previous work on problems with exogenous uncertainty can be found in Sahinidis (2004), Schultz (2003) and Birge (1997).

In general, project decisions can influence the stochastic process in at least two ways. On one hand, the decision-maker may cause alteration of the probability distribution by making one possibility more likely than the other. On the other hand, the decision-maker may not directly affect the probability distributions but could act to get more accurate information by resolving the uncertainty (partially). The difference is that while in the first case the decision-maker can force one possibility to become more probable, in the second case the decision-maker can only become more sure as to which possibility may occur in future.

Viswanath et al. (2004) address an instance of the first type of endogenous uncertainty where optimization decisions can influence the probability distribution. They consider a two-stage network traversal problem where each arc is associated with a probability that represents the probability that the arc will be available for traversal after some disaster. In the first stage, investments are made to increase the probabilities associated with some of the arcs. This is followed by a random event which renders some of the arcs unavailable for traversal. In the second stage, a path from the source to the destination has to be traversed using the available arcs. The aim is to choose the arcs for investment such that the expected shortest path length from the source to the destination is minimized. This problem arises in planning disaster relief between cities with the possibility that some of the inter-connecting routes may become unusable due to the disaster.

Ahmed (2000) presents more examples relating to network design, server selection and facility location where the decision-maker can influence the probability distributions. The author presents a 0-1 hyperbolic programming formulation and an exact solution algorithm for single stage problems with discrete decisions.

The gas field development planning problem is a real world example of the second type of endogenous uncertainty where the optimization decisions give more accurate information by resolving the uncertainty. In this problem, a set of fields (reservoirs of gas) are available for production. The size and quality of the reserves of these fields are uncertain. The uncertainty in a field will be resolved only when a facility is installed at the field. Thus, the investment decisions control when the uncertainty will be resolved. Therefore, apart from considering the large capital expenditures (over US $\$ 100$ Million) and revenues associated with investment at a field, it is also important to consider the potential of obtaining valuable information as a result of the investment. This information could lead to "better" decisions in the future.

A similar problem is the capacity expansion of process networks under yield uncertainty where an 
existing network of processing units can be expanded by installing units that are based on new technology. The yields (or productivities) of these units are uncertain and the uncertainty in a unit is resolved only after the unit is installed and operated in the existing conditions. Thus, the investment decisions determine when the uncertainty will be resolved. We use this problem in section 9 to illustrate that when the value of information is sufficiently high, it may be optimal for the decision-maker to first resolve the uncertainty by making small investments and then make higher investments based on the observations.

Another instance of this type of endogenous uncertainty arises in the multistage network interdiction problem. In each stage, the interdictor interdicts some of the nodes followed by which the operator tries to traverse the network along the shortest path. The exact network structure is unknown to the interdictor, but various possibilities are postulated through a set of scenarios. In each stage, the uncertainty is (partially) resolved based on the path taken by the operator, which is implicitly determined by the interdiction decisions. Thus, the aim of the interdictor is to interdict the nodes such that the most "valuable" information is obtained and the objective maximized.

Jonsbraten et al. (1998) first addressed problems with endogenous uncertainty where project decisions give more accurate information by resolving the uncertainty. The authors present an implicit enumeration based branch and bound algorithm for this class of problems. Results for two-stage problems are also presented. Held and Woodruff (2003) present heuristic solution methods for the multistage network interdiction problem. Both these papers assume that every resolution of uncertainty excludes at least one realization or scenario from the set of future possibilities. Jonsbraten (1998) addresses a variant of the oil (or gas) field problem where investment decisions lead to resolution of uncertainty but none of the scenarios may be excluded from the set of future possibilities. The author proposes an implicit enumeration algorithm where the resolution of uncertainty is modeled using a Bayesian approach.

Goel and Grossmann (2004) used the gas field problem to illustrate an approach for formulating rigorous stochastic programs for problems where the decisions give more accurate information by resolving the uncertainty. In this approach, the interaction between the decisions and the resolution of uncertainty is captured through a disjunctive formulation of the non-anticipativity constraints. The authors also present a heuristic algorithm to solve the gas field problem.

In this paper, we generalize the above approach to problems that have both exogenous and endogenous uncertainties. We consider the second type of endogenous uncertainty where the project decisions lead to resolution of uncertainty. This paper is organized as follows. In section 2 we present a brief background on stochastic programming formulations with exogenous uncertainty. In section 3 we present the manufacturing related "sizes problem" to motivate the class of problems being considered. Next we present a generic description of the broad class of problems under consideration. Sections 5 and 6 explain the notation and the proposed stochastic program, respectively. 


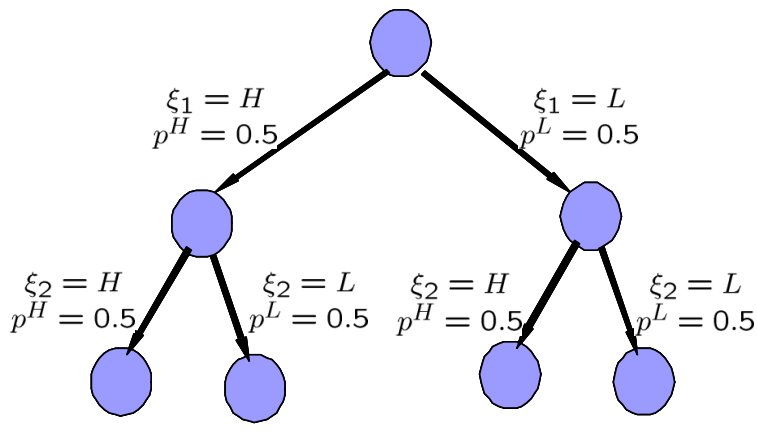

(a) Standard scenario tree

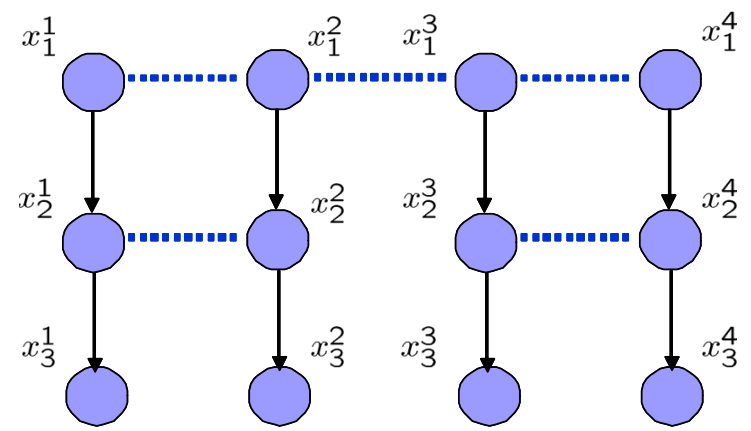

(b) Reformulated scenario tree with variables $x_{t}^{s}$

Figure 1: Equivalent scenario trees

Section 7 presents theoretical properties that lead to reduction in the dimensionality of the model. In section 8 we present a branch and bound algorithm based on Lagrangean duality to solve the proposed model. Finally, section 9 presents results to illustrate the advantages of our approach.

\section{Background}

We restrict the scope of this paper to problems where the uncertainty can be represented by discrete probability distributions and the time horizon is represented by a discrete set of time periods. For such problems, the stochastic process can be represented by a scenario tree, where each node represents a possible information state. An arc emanating from a node for time period $t$ represents a possible transition to a node for time period $t+1$. The probability associated with an arc represents the probability of transition along that arc. Multiple arcs emanating from a node for time period $t$ represent multiple possibilities for transition and hence, that uncertainty in some parameter(s) will be resolved at the end of time period $t$. In a scenario tree, a path from the root node to a leaf node represents a scenario. Physically, a scenario represents one possible combination of values for all uncertain parameters. The probability of a scenario is the probability of reaching the corresponding leaf node from the root node.

Fig. 1(a) represents the scenario tree for a problem with two uncertain parameters $\xi_{1}, \xi_{2}$ and three time periods. Possible realizations for both parameters include $H$ ("High") and $L$ ("Low") where both realizations are equally probable. The uncertainties in $\xi_{1}$ and $\xi_{2}$ are resolved after the first and second time periods, respectively. The scenario tree has four scenarios, each with probability equal to 0.25 .

Ruszczynski (1997) illustrates an alternative representation of scenario trees where each scenario is represented by a set of unique nodes (Fig. 1(b)). If the nodes for scenarios $s, s^{\prime}$ in time period 
$t$ correspond to the same information state (represented by horizontal dotted lines linking the nodes in Fig. 1(b)), the two scenarios are said to be indistinguishable in time period $t$. In general, scenarios $s, s^{\prime}$ are indistinguishable in time period $t$ if the two scenarios are identical in realizations for all uncertain parameters in which uncertainty has been resolved up till time $t$. The concept of indistinguishability is central to the non-anticipativity based approach to stochastic programming.

(SSP) is a "standard" stochastic program (Jonsbraten et al. (1998)) for a linear problem with $T$ time periods and scenario tree $\mathcal{S}$.

$$
\begin{aligned}
(\mathrm{SSP}) \min \sum_{s} p^{s} \sum_{t} c_{t}^{s} x_{t}^{s} & \\
\text { s.t. } \quad \sum_{\tau \leq t} A_{\tau, t}^{s} x_{\tau}^{s} & \leq a_{t}^{s} \quad \forall(t, s) \\
x_{t}^{s} & \in \mathcal{X}_{t}^{s} \quad \forall(t, s) \\
x_{t}^{s} & =x_{t}^{s^{\prime}} \quad \forall\left(s, s^{\prime}, t\right) \in \mathcal{N}_{\mathcal{S}}^{e}
\end{aligned}
$$

Parameter $p^{s}$ represents the probability of scenario $s$ while variables $x_{t}^{s}$ represent decision variables for time period $t$ in scenario $s$. (1a) represents the objective of minimizing the expectation of some economic criterion. Constraint (1b) represents single-period and period-linking constraints for a particular scenario which are characteristic of any multi-period model. Constraint (1c) represents integrality and bound restrictions on variables $x_{t}^{s}$. $\mathcal{N}_{\mathcal{S}}^{e}$ represents the set of tuples $\left(s, s^{\prime}, t\right)$ such that scenarios $s$ and $s^{\prime}$ are indistinguishable in time period $t$ for scenario tree $\mathcal{S}$. The non-anticipativity or implementability constraints (1d) link decisions for different scenarios. These constraints state that if scenarios $s, s^{\prime}$ are indistinguishable in time period $t$ then decisions for $s, s^{\prime}$ in $t$ should be the same. In other words, decisions cannot be based on knowledge that will be revealed in the future.

When the uncertainty is of exogenous nature, the probabilities $p^{s}$ and the set $\mathcal{N}_{\mathcal{S}}^{e}$ are independent of the optimization variables. Thus, these are inputs to the optimization model. However, if the optimization decisions can influence the probability distribution, then probabilities $p^{s}$ have to be treated as optimization variables. On the other hand, if the optimization decisions influence the resolution of uncertainty, then the scenario tree and hence the set $\mathcal{N}_{\mathcal{S}}^{e}$ depends on the decisions (Jonsbraten et al. (1998), Goel and Grossmann (2004)). We generalize the approach of Goel and Grossmann (2004) to problems with both exogenous and endogenous uncertainty by formulating the inter-dependence of $\mathcal{N}_{\mathcal{S}}^{e}$ and the optimization variables as a disjunctive program.

\section{Motivating Example}

The sizes problem (Jonsbraten et al. (1998), Jorjani et al. (1999)) is a specific example of the class of problems under consideration. In this problem, a production line has to meet the demand 
for a product in a set of different sizes, $\mathcal{I}=\{1,2, \ldots, I\}$, in each time period of a time horizon $\mathcal{T}=\{1,2, \ldots, T\}$. If the demand for a size cannot be met, the deficit can be filled by the delivery of a bigger size. However, this involves a substitution cost. Other costs include fixed production costs for set-up of equipment for each size produced in each time period, variable inventory costs and variable production costs for each unit produced.

The demands, represented by $\xi_{t}$ for time period $t \in \mathcal{T}$, are uncertain. The variable costs of production, represented by $\theta_{i}$ for size $i \in \mathcal{I}$, remain constant over the time horizon but are also uncertain. The demand in time period $t$ will be observed automatically in that time period. On the other hand, the uncertainty in variable production cost for size $i, \theta_{i}$, will be resolved only when that size is produced for the first time. Thus, demand uncertainty is exogenous while the uncertainty in variable production costs is endogenous.

Decisions to be made in each time period include whether to produce size $i$ or not (binary variables $b_{i, t}$ ), number of units of size $i$ to be produced (variables $y_{i, t}$ ) and number of units of size $i$ to be used to satisfy demands of size $i^{\prime}$ (variables $\left.x_{i, i^{\prime}, t}\right)$. Production decisions $\left(b_{i, t}, y_{t}\right)$ are implemented at the beginning of time period $t$. Then uncertainty is resolved in demands for time period $t$ and in variable production costs for sizes produced for the first time in time period $t$. Finally, substitution decisions $\left(x_{i, i^{\prime}, t}\right)$ are implemented to satisfy demands for time period $t$.

\section{Generic problem description}

In the class of problems under consideration, the time horizon is represented by the discrete set of time periods $\mathcal{T}=\{1,2, \ldots, T\}$. $\xi_{t}$ represents the vector of exogenous uncertain parameters associated with time period $t \in \mathcal{T}$. The uncertainty in $\xi_{t}$ will be resolved automatically in time period $t . \Xi$ represents the discrete set of possible realizations for vector $\xi=\left(\xi_{1}, \xi_{2}, \ldots, \xi_{T}\right)$.

Set $\mathcal{I}=\{1,2, \ldots, I\}$ represents the set of "sources" of endogenous uncertainty while $\theta_{i}$ represents the endogenous uncertain parameter associated with source $i \in \mathcal{I}$. The discrete set of possible realizations for $\theta_{i}$ is represented by $\Theta_{i}$. The resolution of uncertainty in $\theta_{i}$ depends on binary decision variables $b_{i, t}$. Specifically, the uncertainty in $\theta_{i}$ will be resolved in time period $t$ if binary decision $b_{i, t}=1$ and $b_{i, \tau}=0 \quad \forall \tau<t$. Besides decisions represented by variables $b_{i, t}$, other decisions to be made in time period $t$ are represented by variables $y_{t}$ and $x_{t}$ together.

The sequence of events in each time period is as follows. Decisions $y_{t}$ and $b_{i, t}$ are implemented at the beginning of time period $t$. This is followed by resolution of uncertainty in the exogenous parameters $\xi_{t}$ and in the endogenous parameter $\theta_{i}$ for source $i$ if $b_{i, t}=1$ and $b_{i, \tau}=0 \quad \forall \tau<t$. Finally, decisions $x_{t}$ are implemented at the end of the time period. 
In general, variables $b_{i, t}$ may represent investment or operation decisions associated with source $i$. In the sizes problem, variables $b_{i, t}$ represent whether size $i$ is produced in time period $t$ or not. In the gas field problem considered by Goel and Grossmann (2004), these variables represent whether or not investment is made at field $i$ in time period $t$. The uncertainty associated with a size or a field is resolved in time period $t$ if the production of that size or the investment at that field is carried out for the first time in time period $t$.

Note that for ease of exposition, we assume that there is only one endogenous uncertain parameter associated with source $i$ for all $i \in \mathcal{I}$. Thus, we assume that $\theta_{i}$ is a scalar for all $i \in \mathcal{I}$. At the end of section 7 we describe how our approach extends to the more general case where $\theta_{i}$ may be a vector for some $i \in \mathcal{I}$.

\section{Notations and definitions}

In order to make the following discussion more comprehensible, we first explain the notation and definitions used in this paper. Each scenario in this problem corresponds to one possible realization for the vector $\left(\xi_{1}, \xi_{2}, \ldots, \xi_{T}, \theta_{1}, \theta_{2}, \ldots, \theta_{I}\right)$. We assume that the set of scenarios corresponds to $\Xi \times\left(\times_{i \in \mathcal{I}} \Theta_{i}\right)$, i.e., for any realization of the vector of exogenous parameters, $\xi=\left(\xi_{1}, \xi_{2}, \ldots, \xi_{T}\right)$, the set of scenarios includes scenarios corresponding to all possible combinations of realizations for the endogenous parameters. Individual scenarios are indexed as $s \in \mathcal{S}$, where $\mathcal{S}=\{1,2, \ldots, S\}$ represents the set of indices corresponding to all the scenarios. Note that we will use index $s$ to refer to the corresponding scenario. Further, $\theta_{i}^{s}$ and $\xi_{t}^{s}$ will represent the realizations of $\theta_{i}$ and $\xi_{t}$ respectively, in scenario $s$.

For scenarios $s, s^{\prime} \in \mathcal{S}$, the set $\mathcal{D}\left(s, s^{\prime}\right)=\left\{i \mid i \in \mathcal{I}, \theta_{i}^{s} \neq \theta_{i}^{s^{\prime}}\right\}$ represents the set of sources of endogenous uncertainty that distinguish scenarios $s$ and $s^{\prime} .\left|\mathcal{D}\left(s, s^{\prime}\right)\right|$ represents the cardinality of this set. In general, $0 \leq\left|\mathcal{D}\left(s, s^{\prime}\right)\right| \leq I$ holds for all $s, s^{\prime} \in \mathcal{S}$, where $I$ is the number of sources of endogenous uncertainty. By definition, $\mathcal{D}\left(s, s^{\prime}\right)=\mathcal{D}\left(s^{\prime}, s\right)$.

For scenarios $s, s^{\prime} \in \mathcal{S}, \mathbf{t}\left(s, s^{\prime}\right)$ is the latest time period $t$ such that realizations of all exogenous parameters resolved up till and including $t$ are the same in scenarios $s, s^{\prime}$. In other words, $\mathbf{t}\left(s, s^{\prime}\right)$ is the last time period at the end of which scenarios $s$ and $s^{\prime}$ are indistinguishable based on exogenous uncertainty resolved. Mathematically,

$$
\mathbf{t}\left(s, s^{\prime}\right)=\max _{t}\left\{t \mid t \in \mathcal{T}, \xi_{\tau}^{s}=\xi_{\tau}^{s^{\prime}} \forall \tau \in \mathcal{T}, \tau \leq t\right\}
$$

If $\left\{t \mid t \in \mathcal{T}, \xi_{\tau}^{s}=\xi_{\tau}^{s^{\prime}} \forall \tau \in \mathcal{T}, \tau \leq t\right\}=\emptyset$, then we define $\mathbf{t}\left(s, s^{\prime}\right)=0$. Note that there cannot be distinct scenarios $s, s^{\prime} \in \mathcal{S}$ such that $\left|\mathcal{D}\left(s, s^{\prime}\right)\right|=0$ and $\mathbf{t}\left(s, s^{\prime}\right)=T$. This is because if $s, s^{\prime}$ satisfied the above conditions then they would be completely identical. By definition, $\mathbf{t}\left(s, s^{\prime}\right)=\mathbf{t}\left(s^{\prime}, s\right)$. 
$\mathcal{L}^{0}=\left\{\left(s, s^{\prime}\right)\left|s, s^{\prime} \in \mathcal{S}, s<s^{\prime},\right| \mathcal{D}\left(s, s^{\prime}\right) \mid=0\right\}$ represents the set of scenario pairs $\left(s, s^{\prime}\right)$ such that scenarios $s$ and $s^{\prime}$ are identical in terms of realizations for all endogenous parameters. The condition $s<s^{\prime}$ prevents duplicate entries in $\mathcal{L}^{0}$ for the same pair of scenarios $s, s^{\prime}$.

$\mathcal{L}^{1+}=\left\{\left(s, s^{\prime}\right)\left|s, s^{\prime} \in \mathcal{S}, s<s^{\prime},\right| \mathcal{D}\left(s, s^{\prime}\right) \mid \geq 1\right\}$ represents the set of scenario pairs $\left(s, s^{\prime}\right)$ such that $s, s^{\prime}$ differ in realizations of $\theta_{i}$ for at least one $i \in \mathcal{I}$. Also, $\mathcal{L}^{1}=\left\{\left(s, s^{\prime}\right)\left|s, s^{\prime} \in \mathcal{S}, s<s^{\prime},\right| \mathcal{D}\left(s, s^{\prime}\right) \mid=1\right\}$.

$\mathcal{L}_{T}^{1}=\left\{\left(s, s^{\prime}\right) \mid\left(s, s^{\prime}\right) \in \mathcal{L}^{1}, \mathbf{t}\left(s, s^{\prime}\right)=T\right\}$ is the set of scenario pairs $\left(s, s^{\prime}\right)$ such that scenarios $s, s^{\prime}$ differ in the realization of only one endogenous parameter and are identical in realizations for all exogenous parameters.

\section{Model}

In this section we introduce $P 1$, the declarative form of stochastic programs for the class of problems described in section 4 .

$$
\begin{aligned}
& \text { (P1) } \phi=\min \sum_{s \in \mathcal{S}} p^{s} \sum_{t \in \mathcal{T}}\left({ }^{w} c_{t}^{s} w_{t}^{s}+{ }^{x} c_{t}^{s} x_{t}^{s}+{ }^{y} c_{t}^{s} y_{t}^{s}+\sum_{i \in \mathcal{I}}{ }^{b} c_{i, t}^{s} b_{i, t}^{s}\right) \\
& \text { s.t. } \sum_{\substack{\tau \in \mathcal{T} \\
\tau \leq t}}\left({ }^{w} A_{\tau, t}^{s} w_{\tau}^{s}+{ }^{x} A_{\tau, t}^{s} x_{\tau}^{s}+{ }^{y} A_{\tau, t}^{s} y_{\tau}^{s}+\sum_{i \in \mathcal{I}}{ }^{b} A_{i, \tau, t}^{s} b_{i, \tau}^{s}\right) \leq a_{t}^{s} \quad \forall s \in \mathcal{S}, t \in \mathcal{T} \\
& b_{i, 1}^{s}=b_{i, 1}^{s^{\prime}} \quad \forall s, s^{\prime} \in \mathcal{S}, s<s^{\prime}, i \in \mathcal{I} \\
& y_{1}^{s}=y_{1}^{s^{\prime}} \quad \forall s, s^{\prime} \in \mathcal{S}, s<s^{\prime} \\
& x_{t}^{s} \quad=x_{t}^{s^{\prime}} \quad \forall\left(s, s^{\prime}\right) \in \mathcal{L}^{0}, t \in \mathcal{T}, t \leq \mathbf{t}\left(s, s^{\prime}\right) \\
& b_{i, t+1}^{s}=b_{i, t+1}^{s^{\prime}} \quad \forall\left(s, s^{\prime}\right) \in \mathcal{L}^{0}, t \in \mathcal{T}, t \leq \mathbf{t}\left(s, s^{\prime}\right), i \in \mathcal{I} \\
& y_{t+1}^{s}=y_{t+1}^{s^{\prime}} \quad \forall\left(s, s^{\prime}\right) \in \mathcal{L}^{0}, t \in \mathcal{T}, t \leq \mathbf{t}\left(s, s^{\prime}\right) \\
& Z_{t}^{s, s^{\prime}} \Leftrightarrow \bigwedge_{i \in \mathcal{D}\left(s, s^{\prime}\right)}\left[\bigwedge_{\tau=1}^{t}\left(\neg b_{i, \tau}^{s}\right)\right] \quad \forall\left(s, s^{\prime}\right) \in \mathcal{L}^{1+}, t \in \mathcal{T}, t \leq \mathbf{t}\left(s, s^{\prime}\right) \\
& Z_{t}^{s, s^{\prime}} \Leftrightarrow \bigwedge_{i \in \mathcal{D}\left(s, s^{\prime}\right)}\left[\bigwedge_{\tau=1}^{t}\left(\neg b_{i, \tau}^{s^{\prime}}\right)\right] \quad \forall\left(s, s^{\prime}\right) \in \mathcal{L}^{1+}, t \in \mathcal{T}, t \leq \mathbf{t}\left(s, s^{\prime}\right)
\end{aligned}
$$




$$
\begin{array}{cl}
w_{t}^{s} \in \mathcal{W}_{t}^{s}, x_{t}^{s} \in \mathcal{X}_{t}^{s}, y_{t}^{s} \in \mathcal{Y}_{t}^{s}, b_{i, t}^{s} \in\{0,1\} & \forall s \in \mathcal{S}, t \in \mathcal{T}, i \in \mathcal{I} \\
Z_{t}^{s, s^{\prime}} \in\{\text { True }, \text { False }\} & \forall\left(s, s^{\prime}\right) \in \mathcal{L}^{1+}, t \in \mathcal{T}, t \leq \mathbf{t}\left(s, s^{\prime}\right)
\end{array}
$$

In $P 1$, variables $b_{i, t}^{s}, x_{t}^{s}$ and $y_{t}^{s}$ represent the decisions to be made in time period $t$ of scenario $s$. Vector $w_{t}^{s}$ represents the other variables associated with time period $t$ in scenario $s$. In process control terminology, $b_{i, t}^{s}, x_{t}^{s}$ and $y_{t}^{s}$ are "control variables" while $w_{t}^{s}$ are "state variables". $b_{i, t}^{s}$ are binary variables while $x_{t}^{s}$ and $y_{t}^{s}$ are variable vectors that may have both integer and continuous components. As explained in section 4 , decisions $y_{t}^{s}$ and $b_{i, t}^{s}$ are implemented at the beginning of time period $t$, while decisions $x_{t}^{s}$ are implemented at the end of the time period after the resolution of uncertainty in that time period.

The realizations of the cost coefficients of variables $b_{i, t}^{(\cdot)}, x_{t}^{(\cdot)}, y_{t}^{(\cdot)}$ and $w_{t}^{(\cdot)}$ in scenario $s$ are represented by ${ }^{b} c_{i, t}^{s},{ }^{x} c_{t}^{s},{ }^{y} c_{t}^{s}$ and ${ }^{w} c_{t}^{s}$, respectively. Similarly, ${ }^{b} A_{i, \tau, t}^{s},{ }^{x} A_{\tau, t}^{s},{ }^{y} A_{\tau, t}^{s}$ and ${ }^{w} A_{\tau, t}^{s}$ represent the realizations of the constraint coefficient matrices (or vectors) of these variables in scenario $s$. (2) represents the objective of minimizing the expectation of an economic criterion. (3) represents single-period and period-linking constraints for a particular scenario. These include the square system of equality constraints which can be used to eliminate "state" variables $w_{t}^{s}$.

Decisions for different scenarios are linked by non-anticipativity constraints, (4)-(8). The nonanticipativity rule requires that if scenarios $s$ and $s^{\prime}$ are indistinguishable at some time, then decisions in scenarios $s$ and $s^{\prime}$ should be the same at that time. Based on the sequence of events described in section 4 , uncertainty is resolved in time period $t$ after the implementation of decisions $y_{t}^{s}$ and $b_{i, t}^{s}$. Thus, if scenarios $s, s^{\prime}$ are indistinguishable after resolution of uncertainty in time period $t$, then decisions $x_{t}^{(\cdot)}, b_{i, t+1}^{(\cdot)}$ and $y_{t+1}^{(\cdot)}$ should be the same for scenarios $s, s^{\prime}$. Note that in this paper, we refer to the "indistinguishability of the two scenarios after the resolution of exogenous and endogenous uncertainty in time period $t$ " simply by the "indistinguishability of two scenarios in time period $t$ ".

Based on the sequence of events in each time period, all scenarios are indistinguishable before decisions $b_{i, t}^{s}$ and $y_{t}^{s}$ are implemented in the first time period. Thus, decisions $b_{i, 1}^{(\cdot)}$ and $y_{1}^{(\cdot)}$ have to be the same for all scenarios (4). Note that the condition $s<s^{\prime}$ is imposed to avoid duplication of constraints (4) for the same pair of scenarios $s, s^{\prime}$.

(5) represents non-anticipativity constraints linking scenarios $s, s^{\prime}$ such that $\left(s, s^{\prime}\right) \in \mathcal{L}^{0}$; i.e., the realizations of all endogenous parameters in scenarios $s$ and $s^{\prime}$ are identical. In this case, scenarios $s, s^{\prime}$ will be indistinguishable in time period $t$ if and only if these scenarios are identical in realizations of all exogenous parameters observed up till and including time period $t$. Accordingly, (5) applies non-anticipativity constraints on decisions $x_{t}^{(\cdot)}, y_{t+1}^{(\cdot)}, b_{i, t+1}^{(\cdot)}$ for scenarios $s, s^{\prime}$ only if $t$ satisfies $t \leq \mathbf{t}\left(s, s^{\prime}\right)$.

(6)-(8) are non-anticipativity constraints linking scenarios $s, s^{\prime}$ such that $\left(s, s^{\prime}\right) \in \mathcal{L}^{1+}$; i.e., sce- 
narios $s$ and $s^{\prime}$ differ in the realization of at least one endogenous parameter. In this case, the indistinguishability of scenarios $s, s^{\prime}$ in time period $t$ depends on both, endogenous and exogenous uncertainty resolved in the past. Boolean variable $Z_{t}^{s, s^{\prime}}$ is True if and only if scenarios $s$ and $s^{\prime}$ are indistinguishable (after the resolution of uncertainty) in time period $t$. Clearly, for $t>\mathbf{t}\left(s, s^{\prime}\right)$ scenarios $s, s^{\prime}$ can be distinguished simply based on realizations of the exogenous parameters. Hence, $Z_{t}^{s, s^{\prime}}=$ False for $t>\mathbf{t}\left(s, s^{\prime}\right)$. Therefore constraints (6)-(8) are applied only for $t$ such that $t \leq \mathbf{t}\left(s, s^{\prime}\right)$, where $\left(s, s^{\prime}\right) \in \mathcal{L}^{1+}$.

Disjunction (6) imposes the non-anticipativity constraints on variables $x_{t}^{(\cdot)}, y_{t+1}^{(\cdot)}, b_{i, t+1}^{(\cdot)}$ for scenarios $s, s^{\prime}$ only if $Z_{t}^{s, s^{\prime}}$ is True, i.e., if scenarios $s$ and $s^{\prime}$ are indistinguishable in time period $t$. By definition of $\mathbf{t}\left(s, s^{\prime}\right)$, if $t \leq \mathbf{t}\left(s, s^{\prime}\right)$ then the indistinguishability of scenarios $s, s^{\prime}$ in time period $t$ depends purely on the endogenous uncertainty resolved through the decisions. Logic constraints (7) and (8) relate the indistinguishability of scenarios $s, s^{\prime}$ in time period $t$ with decisions $b_{i, \tau}^{s}$ and $b_{i, \tau}^{s^{\prime}}$ respectively. Scenarios $s, s^{\prime}$ differ in realizations of a finite set of endogenous parameters. Constraint (7) states $^{1}$ that $Z_{t}^{s, s^{\prime}}$ is True if and only if uncertainty has not been resolved in any of these parameters up till (and including) time period $t$ of scenario $s$. Similarly, (8) relates variables $Z_{t}^{s, s^{\prime}}$ to the corresponding decision variables for scenario $s^{\prime}$.

Note that to account for the offset in the time index of these variables, the non-anticipativity constraints on variables $b_{i, t+1}^{(\cdot)}, y_{t+1}^{(\cdot)}$ for scenarios $s, s^{\prime}$ are applied only if $t \leq T-1$. Although it may seem that a similar restriction is needed in (5b)-(5c), however, as explained earlier in this section, we cannot have distinct scenarios $s, s^{\prime} \in \mathcal{S}$ such that $\left(s, s^{\prime}\right) \in \mathcal{L}^{0}$ and $\mathbf{t}\left(s, s^{\prime}\right)=T$. Hence, the condition that $t \leq T-1$ is implicit in the condition $t \leq \mathbf{t}\left(s, s^{\prime}\right)$ in $(5 \mathrm{~b})-(5 \mathrm{c})$.

$\mathcal{W}_{t}^{s}, \mathcal{X}_{t}^{s}$ and $\mathcal{Y}_{t}^{s}$ represent the bounds and integrality restrictions on variables $w_{t}^{s}, x_{t}^{s}$ and $y_{t}^{s}$ respectively, for all $t \in \mathcal{T}, s \in \mathcal{S}$.

\section{$7 \quad$ Model properties}

In this section, we present a set of properties that lead to reduction in the dimensionality of the proposed model. Note that we will use $b^{s}$ to represent the vector of variables $b_{i, t}^{s}$ for all $(i, t)$. Similarly, vector $b$ will represent the vector of $b^{s}$ for all $s$. The same convention will be used to represent vectors of variables $w_{t}^{s}, x_{t}^{s}, y_{t}^{s}, Z_{t}^{s, s^{\prime}}$ and parameters introduced later in the paper. The tuple $(b, w, x, y, Z)$ will be used to represent a solution to the model under consideration. Further, in all properties presented in this paper, it is assumed that variables $b_{i, t}^{s} \in\{0,1\}$ and

\footnotetext{
${ }^{1}$ In theory, the logical operator " $\neg$ " should only be used with Boolean variables. Since $b_{i, t}^{s}$ are binary variables, therefore constraints (7) and (8) involve a slight inconsistency in notation. A more rigorous formulation can be obtained at the expense of additional notation by defining (7) and (8) in terms of Boolean variables $B_{i, t}^{s}$ and specifying an equivalence between variables $B_{i, t}^{s}$ and $b_{i, t}^{s}$.
} 
$Z_{t}^{s, s^{\prime}} \in\{$ True, False $\}$. Similarly, solutions $\hat{b}_{i, t}^{s} \in\{0,1\}$ and $\hat{Z}_{t}^{s, s^{\prime}} \in\{$ True, False $\}$.

Proposition 1. Consider constraints (9)-(11) for given $s, s^{\prime}, \hat{t}$ where $s, s^{\prime} \in \mathcal{S}, \hat{t} \in \mathcal{T}, \hat{t} \leq T-1$.

$$
\begin{gathered}
b_{i, 1}^{s}=b_{i, 1}^{s^{\prime}} \quad \forall i \in \mathcal{I} \\
{\left[\begin{array}{c}
Z_{t}^{s, s^{\prime}} \\
b_{i, t+1}^{s}=b_{i, t+1}^{s^{\prime}} \forall i \in \mathcal{I}
\end{array}\right] \vee\left[\neg Z_{t}^{s, s^{\prime}}\right] \quad \forall t \in \mathcal{T}, t \leq \hat{t}} \\
Z_{t}^{s, s^{\prime}} \Leftrightarrow \bigwedge_{i \in \mathcal{D}\left(s, s^{\prime}\right)}\left[\bigwedge_{\tau=1}^{t}\left(\neg b_{i, \tau}^{s}\right)\right] \quad \forall t \in \mathcal{T}, t \leq \hat{t}
\end{gathered}
$$

If vectors $\hat{b}^{s}, \hat{b}^{s^{\prime}}, \hat{Z}^{s, s^{\prime}}$ satisfy (9)-(11) then,

(a) For $t \in \mathcal{T}, t \leq \hat{t}$,

$$
\bigwedge_{i \in \mathcal{D}\left(s, s^{\prime}\right)}\left[\bigwedge_{\tau=1}^{t}\left(\neg \hat{b}_{i, \tau}^{s}\right)\right] \Rightarrow\left[\hat{b}_{i, \tau}^{s}=\hat{b}_{i, \tau}^{s^{\prime}} \quad \forall i \in \mathcal{I}, \tau \in \mathcal{T}, \tau \leq t+1\right]
$$

(b) For $t=\hat{t}+1$,

$$
\bigwedge_{i \in \mathcal{D}\left(s, s^{\prime}\right)}\left[\bigwedge_{\tau=1}^{t}\left(\neg \hat{b}_{i, \tau}^{s}\right)\right] \Rightarrow\left[\hat{b}_{i, \tau}^{s}=\hat{b}_{i, \tau}^{s^{\prime}} \quad \forall i \in \mathcal{I}, \tau \in \mathcal{T}, \tau \leq t\right]
$$

(c) For $t \in \mathcal{T}, t \leq \hat{t}+1$,

$$
\bigwedge_{i \in \mathcal{D}\left(s, s^{\prime}\right)}\left[\bigwedge_{\tau=1}^{t}\left(\neg \hat{b}_{i, \tau}^{s}\right)\right] \Leftrightarrow \bigwedge_{i \in \mathcal{D}\left(s, s^{\prime}\right)}\left[\bigwedge_{\tau=1}^{t}\left(\neg \hat{b}_{i, \tau}^{s^{\prime}}\right)\right]
$$

(d) For $t \in \mathcal{T}, t \leq \hat{t}$,

$$
\bigwedge_{i \in \mathcal{D}\left(s, s^{\prime}\right)}\left[\bigwedge_{\tau=1}^{t}\left(\neg \hat{b}_{i, \tau}^{s^{\prime}}\right)\right] \Rightarrow\left[\hat{b}_{i, \tau}^{s}=\hat{b}_{i, \tau}^{s^{\prime}} \quad \forall i \in \mathcal{I}, \tau \in \mathcal{T}, \tau \leq t+1 .\right]
$$

(e) For $t=\hat{t}+1$,

$$
\bigwedge_{i \in \mathcal{D}\left(s, s^{\prime}\right)}\left[\bigwedge_{\tau=1}^{t}\left(\neg \hat{b}_{i, \tau}^{s^{\prime}}\right)\right] \Rightarrow\left[\hat{b}_{i, \tau}^{s}=\hat{b}_{i, \tau}^{s^{\prime}} \quad \forall i \in \mathcal{I}, \tau \in \mathcal{T}, \tau \leq t .\right]
$$

Note that the left hand sides of (a) and (b) involve variables $b_{i, \tau}^{s}$ while the left hand sides of (d) and (e) involve variables $b_{i, \tau}^{s^{\prime}}$. 
Proof. See Appendix A.

We use Proposition 1 as a basis to prove the following theorem.

Theorem 1. If solution $(\hat{b}, \hat{w}, \hat{x}, \hat{y}, \hat{Z})$ satisfies (4a), (6) and (7), then it also satisfies (8). Thus, constraint (8) is redundant in $P 1$.

Proof. Suppose solution $(\hat{b}, \hat{w}, \hat{x}, \hat{y}, \hat{Z})$ satisfies (4a), (6) and (7). Consider scenarios $s_{a}, s_{b} \in \mathcal{S}$ such that $\left(s_{a}, s_{b}\right) \in \mathcal{L}^{1+}$. We will prove that solution $(\hat{b}, \hat{w}, \hat{x}, \hat{y}, \hat{Z})$ satisfies $(8)$ for $\left(s, s^{\prime}\right)=\left(s_{a}, s_{b}\right)$. The theorem follows as a result.

By definition, $\mathbf{t}\left(s_{a}, s_{b}\right)=\max _{t}\left\{t \mid t \in \mathcal{T}, \xi_{\tau}^{s_{a}}=\xi_{\tau}^{s_{b}} \forall \tau \in \mathcal{T}, \tau \leq t\right\}$. If $\left\{t \mid t \in \mathcal{T}, \xi_{\tau}^{s_{a}}=\xi_{\tau}^{s_{b}} \forall \tau \in\right.$ $\mathcal{T}, \tau \leq t\}=\emptyset$, then by convention $\mathbf{t}\left(s_{a}, s_{b}\right)=0$. Hence, $(\hat{b}, \hat{w}, \hat{x}, \hat{y}, \hat{Z})$ satisfies (8) vacuously for $\left(s, s^{\prime}\right)=\left(s_{a}, s_{b}\right)$.

If $\left\{t \mid t \in \mathcal{T}, \xi_{\tau}^{s_{a}}=\xi_{\tau}^{s_{b}} \forall \tau \in \mathcal{T}, \tau \leq t\right\} \neq \emptyset$, then $\mathbf{t}\left(s_{a}, s_{b}\right) \geq 1$. Since $(\hat{b}, \hat{w}, \hat{x}, \hat{y}, \hat{Z})$ satisfies (4a), (6) and (7) and $\left(s_{a}, s_{b}\right) \in \mathcal{L}^{1+}$, therefore sub-vectors $\hat{b}^{s_{a}}, \hat{b}^{s_{b}}, \hat{Z}^{s_{a}, s_{b}}$ satisfy (9)-(11) for $\left(s, s^{\prime}\right)=\left(s_{a}, s_{b}\right)$, $\hat{t}=\min \left(T-1, \mathbf{t}\left(s_{a}, s_{b}\right)\right)$. (The equality constraint on variables $b_{i, t+1}^{(\cdot)}$ inside (6) is applied only if $t \leq T-1$. Hence, $\left(\hat{b}^{s_{a}}, \hat{b}^{s_{b}}, \hat{Z}^{s_{a}, s_{b}}\right)$ is guaranteed to satisfy (10) only for $\left.t \leq \min \left(T-1, \mathbf{t}\left(s_{a}, s_{b}\right)\right)\right)$.

Using result (c) of Proposition 1, we get

$$
\bigwedge_{i \in \mathcal{D}\left(s_{a}, s_{b}\right)}\left[\bigwedge_{\tau=1}^{t}\left(\neg \hat{b}_{i, \tau}^{s_{a}}\right)\right] \Leftrightarrow \bigwedge_{i \in \mathcal{D}\left(s_{a}, s_{b}\right)}\left[\bigwedge_{\tau=1}^{t}\left(\neg \hat{b}_{i, \tau}^{s_{b}}\right)\right] \quad \forall t \in \mathcal{T}, t \leq \min \left(T-1, \mathbf{t}\left(s_{a}, s_{b}\right)\right)+1
$$

Since $\mathbf{t}\left(s_{a}, s_{b}\right) \leq T$, therefore $\min \left(T-1, \mathbf{t}\left(s_{a}, s_{b}\right)\right)+1=\min \left(T, \mathbf{t}\left(s_{a}, s_{b}\right)+1\right) \geq \mathbf{t}\left(s_{a}, s_{b}\right)$. Hence,

$$
\bigwedge_{i \in \mathcal{D}\left(s_{a}, s_{b}\right)}\left[\bigwedge_{\tau=1}^{t}\left(\neg \hat{b}_{i, \tau}^{s_{a}}\right)\right] \Leftrightarrow \bigwedge_{i \in \mathcal{D}\left(s_{a}, s_{b}\right)}\left[\bigwedge_{\tau=1}^{t}\left(\neg \hat{b}_{i, \tau}^{s_{b}}\right)\right] \quad \forall t \in \mathcal{T}, t \leq \mathbf{t}\left(s_{a}, s_{b}\right)
$$

Since sub-vectors $\hat{b}^{s_{a}}, \hat{b}^{s_{b}}, \hat{Z}^{s_{a}, s_{b}}$ satisfy (7) for $\left(s, s^{\prime}\right)=\left(s_{a}, s_{b}\right)$, we can combine (7) with (12) to infer that sub-vectors $\hat{b}^{s_{a}}, \hat{b}^{s_{b}}, \hat{Z}^{s_{a}, s_{b}}$ satisfy (8). The result follows.

Proposition 2. Consider constraints (13)-(16) in variables $b, x, y, Z$ defined over the tuple $\left(s, s^{\prime}, t\right)$

$$
\begin{aligned}
b_{i, t}^{s} & =b_{i, t}^{s^{\prime}} \quad \forall i \in \mathcal{I} \\
y_{t}^{s} & =y_{t}^{s^{\prime}} \\
x_{t}^{s} & =x_{t}^{s^{\prime}} \\
b_{i, t+1}^{s} & =b_{i, t+1}^{s^{\prime}} \quad \forall i \in \mathcal{I} \\
y_{t+1}^{s} & =y_{t+1}^{s^{\prime}}
\end{aligned}
$$




$$
\begin{aligned}
& {\left[\begin{array}{cccc}
Z_{t}^{s, s^{\prime}} & & \\
x_{t}^{s} & = & x_{t}^{s^{\prime}} & \\
b_{i, t+1}^{s} & = & b_{i, t+1}^{s^{\prime}} \\
y_{t+1}^{s} & = & y_{t+1}^{s^{\prime}} & \text { if } t \leq T-1
\end{array}\right] \vee\left[\neg Z_{t}^{s, s^{\prime}}\right]} \\
& Z_{t}^{s, s^{\prime}} \Leftrightarrow \bigwedge_{i \in \mathcal{D}\left(s, s^{\prime}\right)}\left[\bigwedge_{\tau=1}^{t}\left(\neg b_{i, \tau}^{s}\right)\right]
\end{aligned}
$$

If vectors $\hat{b}, \hat{x}, \hat{y}, \hat{Z}$ satisfy

(i) Constraints (13a)-(13b) for $\left(s, s^{\prime}, t\right)$ such that $s, s^{\prime} \in \mathcal{S}, s<s^{\prime}, t=1$

(ii) Constraint (14) for $\left(s, s^{\prime}, t\right)$ such that $\left(s, s^{\prime}\right) \in \mathcal{L}^{0}, t \in \mathcal{T}, t \leq \boldsymbol{t}\left(s, s^{\prime}\right)$

(iii) Constraints (15)-(16) for $\left(s, s^{\prime}, t\right)$ such that $\left(s, s^{\prime}\right) \in \mathcal{L}_{T}^{1}, t \in \mathcal{T}$

then $\hat{b}, \hat{x}, \hat{y}, \hat{Z}$ satisfy constraints (15)-(16) for $\left(s, s^{\prime}, t\right)$ such that $\left(s, s^{\prime}\right) \in \mathcal{L}^{1+}, t \in \mathcal{T}, t \leq \boldsymbol{t}\left(s, s^{\prime}\right)$.

Proof. See Appendix C (Based on Lemma 1, Appendix B).

Based on the above proposition, we define model $P 2$ where (17) and (18) are applied instead of (6) and (7), respectively. Also, (8) has been dropped.

$(P 2) \quad \phi=\min \sum_{s \in \mathcal{S}} p^{s} \sum_{t \in \mathcal{T}}\left({ }^{w} c_{t}^{s} w_{t}^{s}+{ }^{x} c_{t}^{s} x_{t}^{s}+{ }^{y} c_{t}^{s} y_{t}^{s}+\sum_{i \in \mathcal{I}}{ }^{b} c_{i, t}^{s} b_{i, t}^{s}\right)$

s.t. $(3),(4),(5)$

$$
\begin{array}{lll}
{\left[\begin{array}{lll}
Z_{t}^{s, s^{\prime}} & \\
x_{t}^{s}= & x_{t}^{s^{\prime}} & \\
b_{i, t+1}^{s}= & b_{i, t+1}^{s^{\prime}} \\
y_{t+1}^{s}= & y_{t+1}^{s^{\prime}} & \text { if } t \leq T-1
\end{array}\right]} & \vee\left[\neg Z_{t}^{s, s^{\prime}}\right] \\
& \forall\left(s, s^{\prime}\right) \in \mathcal{L}_{T}^{1}, t \in \mathcal{T} \\
Z_{t}^{s, s^{\prime}} \Leftrightarrow\left[\bigwedge_{\tau=1}^{t}\left(\neg b_{i, \tau}^{s}\right)\right] & \forall\left(s, s^{\prime}\right) \in \mathcal{L}_{T}^{1}, t \in \mathcal{T},\{i\}=\mathcal{D}\left(s, s^{\prime}\right) \\
w_{t}^{s} \in \mathcal{W}_{t}^{s}, x_{t}^{s} \in \mathcal{X}_{t}^{s}, y_{t}^{s} \in \mathcal{Y}_{t}^{s}, b_{i, t}^{s} \in\{0,1\} & \forall s \in \mathcal{S}, t \in \mathcal{T}, i \in \mathcal{I} \\
Z_{t}^{s, s^{\prime}} \in\{\text { True }, \text { False }\} & \forall\left(s, s^{\prime}\right) \in \mathcal{L}_{T}^{1}, t \in \mathcal{T}
\end{array}
$$

Theorem 2. If $(\hat{b}, \hat{w}, \hat{x}, \hat{y}, \hat{Z})$ is an optimal solution P1 then it is also an optimal solution of P2, and vice versa. 
Proof. Since the objective functions of $P 1$ and $P 2$ are the same, it is sufficient to show that the feasible regions of $P 1$ and $P 2$ are the same.

Suppose $(\hat{b}, \hat{w}, \hat{x}, \hat{y}, \hat{Z})$ is a feasible solution of $P 1$. Compare models $P 1$ and $P 2$. Constraints (3)-(5) are common to both models while disjunctions (17) and (6) differ only in the domain for $\left(s, s^{\prime}, t\right)$. Constraint (18) differs from (7) in the domain for $\left(s, s^{\prime}, t\right)$ and in the right hand side of the logic relationship.

Let $\mathcal{F}_{1}$ denote the domain of $\left(s, s^{\prime}, t\right)$ in $(6)$-(7) and let $\mathcal{F}_{2}$ denote the domain of $\left(s, s^{\prime}, t\right)$ in $(17)$-(18). Thus,

$$
\begin{gathered}
\mathcal{F}_{1}=\left\{\left(s, s^{\prime}, t\right) \mid\left(s, s^{\prime}\right) \in \mathcal{L}^{1+}, t \in \mathcal{T}, t \leq \mathbf{t}\left(s, s^{\prime}\right)\right\} \\
\mathcal{F}_{2}=\left\{\left(s, s^{\prime}, t\right) \mid\left(s, s^{\prime}\right) \in \mathcal{L}_{T}^{1}, t \in \mathcal{T}\right\} .
\end{gathered}
$$

where,

$$
\mathcal{L}_{T}^{1}=\left\{\left(s, s^{\prime}\right) \mid\left(s, s^{\prime}\right) \in \mathcal{L}^{1}, \mathbf{t}\left(s, s^{\prime}\right)=T\right\}
$$

By definition,

$$
\mathcal{L}_{T}^{1} \subseteq \mathcal{L}^{1} \subseteq \mathcal{L}^{1+}
$$

Now,

$$
\begin{aligned}
& \mathcal{F}_{2}=\left\{\left(s, s^{\prime}, t\right) \mid\left(s, s^{\prime}\right) \in \mathcal{L}_{T}^{1}, t \in \mathcal{T}\right\} \\
& \equiv\left\{\left(s, s^{\prime}, t\right) \mid\left(s, s^{\prime}\right) \in \mathcal{L}_{T}^{1}, t \in \mathcal{T}, t \leq \mathbf{t}\left(s, s^{\prime}\right)\right\} \quad\left(\text { since } \mathbf{t}\left(s, s^{\prime}\right)=T \text { for }\left(s, s^{\prime}\right) \in \mathcal{L}_{T}^{1}\right) \\
& \subseteq\left\{\left(s, s^{\prime}, t\right) \mid\left(s, s^{\prime}\right) \in \mathcal{L}^{1+}, t \in \mathcal{T}, t \leq \mathbf{t}\left(s, s^{\prime}\right)\right\} \quad\left(\text { since } \mathcal{L}_{T}^{1} \subseteq \mathcal{L}^{1+}\right) \\
& =\mathcal{F}_{1}
\end{aligned}
$$

Also, $\left|\mathcal{D}\left(s, s^{\prime}\right)\right|=1$ for $\left(s, s^{\prime}, t\right) \in \mathcal{F}_{2}$. Therefore, the right hand side of (7) reduces to the right hand side of (18). Since $\mathcal{F}_{2} \subseteq \mathcal{F}_{1}$, therefore $P 2$ is a relaxation of $P 1$. Thus $(\hat{b}, \hat{w}, \hat{x}, \hat{y}, \hat{Z})$ should be a feasible solution of $P 2$.

Conversely, suppose $(\hat{b}, \hat{w}, \hat{x}, \hat{y}, \hat{Z})$ is a feasible solution of $P 2$. Thus, $(\hat{b}, \hat{w}, \hat{x}, \hat{y}, \hat{Z})$ satisfies $(3)$, (4), (5), (17) and (18). Using Proposition 2 we can infer that $(\hat{b}, \hat{w}, \hat{x}, \hat{y}, \hat{Z})$ satisfies (6) and (7). Further, using Theorem 1 we can infer that $(\hat{b}, \hat{w}, \hat{x}, \hat{y}, \hat{Z})$ satisfies $(8)$. Thus, $(\hat{b}, \hat{w}, \hat{x}, \hat{y}, \hat{Z})$ is a feasible solution of $P 1$.

The following remarks can be made about the proposed model.

1. According to model $P 2$, non-anticipativity constraints need to be applied for scenarios $s$ and $s^{\prime}$ only if the scenarios either differ exclusively in realizations for exogenous uncertain parameters, or differ exclusively in the realization of one endogenous uncertain parameter.

2. The "standard" stochastic programming formulation (1) is clearly a specific case of model P2 when there is only exogenous uncertainty $\left(\mathcal{L}^{1+}=\mathcal{L}_{T}^{1}=\emptyset\right)$. 
3. The proofs of Proposition 1, Theorem 1 and Theorem 2 are independent of the choice of the set of scenarios. To illustrate the dependence of Proposition 2 on the set of scenarios, consider indices $s_{a}, s_{b} \in \mathcal{S}$ such that the corresponding scenarios differ in the realizations of $r$ endogenous parameters, where $r=\left|\mathcal{D}\left(s_{a}, s_{b}\right)\right| \geq 1$. Broadly, Proposition 2, which is used in Theorem 2, is based on the assumption that there exist indices $s_{1}, s_{2}, \ldots, s_{r} \in \mathcal{S}$ such that $\left(s_{a}, s_{1}\right),\left(s_{1}, s_{2}\right),\left(s_{2}, s_{3}\right), \ldots,\left(s_{r-1}, s_{r}\right) \in \mathcal{L}_{T}^{1}$ while $\left(s_{r}, s_{b}\right) \in \mathcal{L}^{0}$. Proposition 2 is then a result of the fact that the non-anticipativity constraints linking $s_{a}$ with $s_{b}$ are implied by the "chaining" of non-anticipativity constraints linking $s_{a}$ with $s_{1}, s_{1}$ with $s_{2}, s_{2}$ with $s_{3}, \ldots$, $s_{r-1}$ with $s_{r}$ and $s_{r}$ with $s_{b}$.

Since we choose the set of scenarios as $\Xi \times\left(\times_{i \in \mathcal{I}} \Theta_{i}\right)$, for any realization of the vector of exogenous parameters $\xi$, the set of scenarios includes all possible combinations of realizations for the endogenous parameters. Thus, we can generate $r$ "intermediate" scenarios from scenario $s_{a}$ by progressively changing the realization of one of the $r$ distinguishing endogenous parameters to the corresponding realization in scenario $s_{b}$. The realizations of all exogenous parameters in these $r$ scenarios are identical to those in $s_{a}$. Since $s_{a} \in \mathcal{S}$, these $r$ scenarios also belong to the set of scenarios. Thus, we can choose indices $s_{1}, s_{2}, \ldots, s_{r} \in \mathcal{S}$ for these $r$ scenarios. Hence, the non-anticipativity constraints for $s_{a}, s_{b}$ follow by "chaining", as explained above.

4. The models and proofs presented here are based on the assumption that the endogenous uncertainty associated with source $i$ can be represented by one parameter. Thus, $\theta_{i}$ is a scalar. To consider the more general case, suppose $\theta_{i}$ is an $n_{i} \times 1$ vector. For example, in the gas field problem, the uncertainty in a field is represented by uncertainty in the size and quality of the field. Therefore, in that problem $n_{i}=2$ for each field $i$.

If we choose the set of scenarios as $\Xi \times\left(\times_{i \in \mathcal{I}} \Theta_{i}\right)$, where $\left(\times_{i \in \mathcal{I}} \Theta_{i}\right)$ represents all possible combinations of realizations for vectors $\theta_{i}$ for all $i$, then we can again use the "chaining" argument to prove that the solutions to models $P 1$ and $P 2$ are the same for

$$
\begin{aligned}
\mathcal{L}_{T}^{1}=\left\{\left(s, s^{\prime}\right) \mid\right. & s, s^{\prime} \in \mathcal{S}, s<s^{\prime}, \mathbf{t}\left(s, s^{\prime}\right)=T, \\
& \exists\left(i^{*}, l^{*}\right), i^{*} \in \mathcal{I}, l^{*} \in\left\{1,2, \ldots, n_{i^{*}}\right\} \text { such that } \\
& \theta_{l^{*}, i^{*}}^{s} \neq \theta_{l^{*}, i^{*}}^{s^{\prime}}, \\
& \left.\theta_{l, i}^{s}=\theta_{l, i}^{s^{\prime}} \forall l \in\left\{1,2, \ldots, n_{i}\right\}, i \in \mathcal{I} \backslash\left\{i^{*}\right\}\right\}
\end{aligned}
$$

However, stronger results may be obtained if the set of scenarios is chosen as $\Xi \times\left(\times_{i \in \mathcal{I}}\left(\times_{l=1}^{n_{i}} \Theta_{l, i}\right)\right)$, where $\Theta_{l, i}$ represents the set of possible realizations for endogenous uncertain parameter $\theta_{l, i}$ associated with source $i$. The "chaining" argument can then be used to prove that the solutions to models $P 1$ and $P 2$ are the same for

$$
\mathcal{L}_{T}^{1} \quad=\left\{\left(s, s^{\prime}\right) \mid \quad s, s^{\prime} \in \mathcal{S}, s<s^{\prime}, \mathbf{t}\left(s, s^{\prime}\right)=T,\right.
$$




$$
\begin{aligned}
& \exists\left(i^{*}, l^{*}\right), i^{*} \in \mathcal{I}, l^{*} \in\left\{1,2, \ldots, n_{i^{*}}\right\} \text { such that } \\
& \theta_{l^{*}, i^{*}}^{s} \neq \theta_{l^{*}, i^{*}}^{s^{\prime}} \\
& \theta_{l, i^{*}}^{s}=\theta_{l, i^{*}}^{s^{\prime}} \forall l \in\left\{1,2, \ldots, n_{i^{*}}\right\} \backslash\left\{l^{*}\right\} \\
& \left.\theta_{l, i}^{s}=\theta_{l, i}^{s^{\prime}} \forall l \in\left\{1,2, \ldots, n_{i}\right\}, i \in \mathcal{I} \backslash\left\{i^{*}\right\}\right\}
\end{aligned}
$$

In other words, the disjunctive non-anticipativity constraints will need to be applied between scenarios $s, s^{\prime}$ only if the two scenarios differ in the realization of exactly one endogenous scalar parameter, $\theta_{l^{*}, i^{*}}$ for some source $i^{*} \in \mathcal{I}$.

5. In the present form, $P 2$ has disjunctions and linear constraints linking Boolean, binary and continuous variables. The model can be reformulated as a mixed integer linear program by representing Boolean variables $Z_{t}^{s, s^{\prime}}$ as $0-1$ variables $z_{t}^{s, s^{\prime}}$ and reformulating the logic constraints and disjunctions as linear constraints using big-M or convex hull reformulations (Balas (1985), Turkay and Grossmann (1996)). It should be noted that if (18) is reformulated as linear constraints, then variables $z_{t}^{s, s^{\prime}}$ will satisfy the integrality condition even if they are represented by continuous variables with bounds $0 \leq z_{t}^{s, s^{\prime}} \leq 1$.

However, solving the MILP reformulation of $P 2$ may be an inefficient approach for large problems. In the next section, we present a specialized branch and bound algorithm motivated by the work of Caroe and Schultz (1999).

\section{Branch and bound algorithm}

Model $P 2$ is coupled in scenarios through the non-anticipativity constraints. In the proposed branch and bound algorithm, lower bounds at each node are generated by solving a Lagrangean dual problem which is obtained by relaxing the non-anticipativity constraints. Each sub-problem in the Lagrangean dual problem corresponds to an MILP for one of the scenarios. An outline of the proposed algorithm is presented in Fig. 2. $\mathcal{P}$ denotes the list of current problems together with the associated lower bounds, $\phi_{R L D}$, while $\phi^{U B}$ represents the objective value of the best feasible solution obtained. The steps of the algorithm are explained in more detail below. For simplicity, we assume that all integer components of variables $x_{t}^{s}$ and $y_{t}^{s}$ correspond to binary variables. 
Step 1 Initialization: $\phi^{U B}=\infty, \mathcal{P}=\left\{P 2_{\text {ref }}\right\}$.

Step 2 Termination: If $\mathcal{P}=\emptyset$, stop. Current best solution is optimal.

Step 3 Node selection: Select and delete $P$ from $\mathcal{P}$. Solve relaxed Lagrangean dual of $P$ to obtain the solution $(\hat{b}, \hat{w}, \hat{x}, \hat{y}, \hat{Z})$ with objective value $\phi_{R L D}(P)$. If the Lagrangean dual is infeasible, go to step 2 . Set $\phi_{L B}=\phi_{R L D}(P)$

Step 4 Bounding: If $\phi_{L B} \geq \phi^{U B}$, go to step 2 (This step can be carried out as soon as the value of the Lagrangean dual goes above $\left.\phi^{U B}\right)$.

Apply heuristic on solution $(\hat{b}, \hat{w}, \hat{x}, \hat{y}, \hat{Z})$ to generate feasible solution $(\bar{b}, \bar{w}, \bar{x}, \bar{y}, \bar{Z})$ with objective value $\bar{\phi}$. If heuristic is successful, let $\phi^{U B}:=\max \left(\phi^{U B}, \bar{\phi}\right)$. Delete from $\mathcal{P}$ all problems $P^{\prime}$ with $\phi_{R L D}\left(P^{\prime}\right) \geq \phi^{U B}$.

If $\quad \phi_{L B} \geq \phi^{U B}, \quad$ go to step 2 .

else, $\quad$ go to step 5 .

Step 5 Branching: Execute step (a) or (b).

(a) On dualized equality constraints: Select $\left(s_{a}, s_{a}^{\prime}, t_{a}\right) \in \mathcal{N}_{P}^{e}$. Create problems $P_{1}, P_{2}$ identical to $P$. Execute branching sub-step.

(b) On relaxed disjunctions: Select $\left(s_{b}, s_{b}^{\prime}, t_{b}\right) \in \mathcal{N}_{P}^{d}$. Create problems $P_{1}, P_{2}, P_{3}$ identical to $P$. Add restrictions $Z_{t_{b}}^{s_{b}, s_{b}^{\prime}}=Z_{t_{b}}^{s_{b}^{\prime}, s_{b}}=$ True to $P_{1}$ and $P_{2}$ and $Z_{t_{b}}^{s_{b}, s_{b}^{\prime}}=Z_{t_{b}}^{s_{b}^{\prime}, s_{b}}=$ False to $P_{3}$, respectively. For $P_{1}$ and $P_{2}$ update $\mathcal{N}_{(\cdot)}^{e}:=\mathcal{N}_{(\cdot)}^{e} \cup\left(s_{b}, s_{b}^{\prime}, t_{b}\right), \mathcal{N}_{(\cdot)}^{d}:=\mathcal{N}_{(\cdot)}^{d} \backslash\left(s_{b}, s_{b}^{\prime}, t_{b}\right)$. Set $\mathcal{N}_{P_{3}}^{d}:=\mathcal{N}_{P_{3}}^{d} \backslash\left(s_{b}, s_{b}^{\prime}, t_{b}\right)$. Add $P_{3}$ to $\mathcal{P}$. Select $\left(s_{a}, s_{a}^{\prime}, t_{a}\right) \in \mathcal{N}_{P_{1}}^{e}$. Execute branching sub-step.

Branching sub-step:

If $\quad t_{a}=0, \quad$ execute (ii) or (iii).

else if $\quad t_{a}=T$, execute (i).

else, $\quad$ execute any one of (i), (ii) and (iii).

(i) Select component $x_{l, t_{a}}$ of $x_{t_{a}}$.

If $x_{l, t_{a}} \in\{0,1\} \quad$ Add bounds $x_{l, t_{a}}^{s_{a}}, x_{l, t_{a}}^{s_{a}^{\prime}} \geq 1$ to $P_{1}$ and $x_{l, t_{a}}^{s_{a}}, x_{l, t_{a}}^{s_{a}^{\prime}} \leq 0$ to $P_{2}$.

else $\quad$ Add bounds $x_{l, t_{a}}^{s_{a}}, x_{l, t_{a}}^{s_{a}^{\prime a}} \geq \tilde{x}_{l, t_{a}}^{s_{a}, s_{a}^{\prime}}$ to $P_{1}$ and $x_{l, t_{a}}^{s_{a}}, x_{l, t_{a}}^{s_{a}^{\prime}} \leq \tilde{x}_{l, t_{a}}^{s_{a}, s_{a}^{\prime}}$ to $P_{2}$.

(ii) Select component $y_{l, t_{a}+1}$ of $y_{t_{a}+1}$.

If $y_{l, t_{a}+1} \in\{0,1\} \quad$ Add bounds $y_{l, t_{a}+1}^{s_{a}}, y_{l, t_{a}+1}^{s_{a}^{\prime}} \geq 1$ to $P_{1}$ and $y_{l, t_{a}+1}^{s_{a}}, y_{l, t_{a}+1}^{s_{a}^{\prime}} \leq 0$ to $P_{2}$.

else $\quad$ Add bounds $y_{l, t_{a}+1}^{s_{a}}, y_{l, t_{a}+1}^{s_{a}^{\prime}} \geq \tilde{y}_{l, t_{a}+1}^{s_{a}, s_{a}^{\prime}}$ to $P_{1}$ and $y_{l, t_{a}+1}^{s_{a}}, y_{l, t_{a}+1}^{s_{a}^{\prime}} \leq \tilde{y}_{l, t_{a}+1}^{s_{a}, s_{a}^{\prime}}$ to $P_{2}$.

(iii) Select variable $b_{i, t_{a}+1}$.

Add bounds $b_{i, t_{a}+1}^{s_{a}}, b_{i, t_{a}+1}^{s_{a}^{\prime}} \geq 1$ to $P_{1}$ and $b_{i, t_{a}+1}^{s_{a}}, b_{i, t_{a}+1}^{s_{a}^{\prime}} \leq 0$ to $P_{2}$.

Add $P_{1}, P_{2}$ to $\mathcal{P}$. Go to step 2 .

Figure 2: Proposed branch and bound algorithm 


$$
\begin{aligned}
& \left(P 2_{\text {ref }}\right) \quad \phi_{\text {ref }}=\min \sum_{s \in \mathcal{S}} p^{s} \sum_{t \in \mathcal{T}}\left({ }^{w} c_{t}^{s} w_{t}^{s}+{ }^{x} c_{t}^{s} x_{t}^{s}+{ }^{y} c_{t}^{s} y_{t}^{s}+\sum_{i \in \mathcal{I}}{ }^{b} c_{i, t}^{s} b_{i, t}^{s}\right) \\
& \text { s.t. }(3),(4),(5),(17),(18) \\
& Z_{t}^{s^{\prime}, s} \Leftrightarrow\left[\bigwedge_{\tau=1}^{t}\left(\neg b_{i, \tau}^{s^{\prime}}\right)\right] \\
& Z_{t}^{s^{\prime}, s}=Z_{t}^{s, s^{\prime}} \\
& \forall\left(s, s^{\prime}\right) \in \mathcal{L}_{T}^{1}, t \in \mathcal{T} \\
& w_{t}^{s} \in \mathcal{W}_{t}^{s}, x_{t}^{s} \in \mathcal{X}_{t}^{s}, y_{t}^{s} \in \mathcal{Y}_{t}^{s}, b_{i, t}^{s} \in\{0,1\} \\
& \forall s \in \mathcal{S}, t \in \mathcal{T}, i \in \mathcal{I} \\
& Z_{t}^{s, s^{\prime}}, Z_{t}^{s^{\prime}, s} \in\{\text { True, False }\} \\
& \forall\left(s, s^{\prime}\right) \in \mathcal{L}_{T}^{1}, t \in \mathcal{T},\{i\}=\mathcal{D}\left(s, s^{\prime}\right) \\
& \forall\left(s, s^{\prime}\right) \in \mathcal{L}_{T}^{1}, t \in \mathcal{T}
\end{aligned}
$$

We first illustrate the formulation of the Lagrangean dual for the problem at the root node. Model $P 2_{\text {ref }}$ is a reformulation of $P 2$ obtained by introducing dummy variables $Z_{t}^{s^{\prime}, s}$ for $\left(s, s^{\prime}\right) \in \mathcal{L}_{T}^{1}$, $t \in \mathcal{T}$. In $P 2_{\text {ref }}$, constraint (19) relates $Z_{t}^{s^{\prime}, s}$ to variables $b^{s^{\prime}}$ while (18) relates $Z_{t}^{s, s^{\prime}}$ to variables $b^{s}$. (20) represents the symmetry restriction on $Z_{t}^{s, s^{\prime}}$ with respect to $s, s^{\prime}$. From the proofs presented in the previous section, it is clear that any solution of model $P 2$ will also satisfy (19) and (20). Therefore, the optimal solutions of $P 2$ and $P 2_{\text {ref }}$ will be the same and hence, $\phi=\phi_{\text {ref }}$. While (19) and (20) are redundant (as shown in Theorem 1), inclusion of these constraints in the model tightens the Lagrangean dual and hence the lower bounds. A qualitative reasoning for the same will be presented later in this section.

Model $P 2_{R L R}$ is obtained from $P 2_{\text {ref }}$ by relaxing disjunctions (17) and replacing equality constraints (4), (5) and (20) by penalty terms in the objective. Parameters ${ }^{b} \lambda_{i, t}^{s, s^{\prime}},{ }^{x} \lambda_{t}^{s, s^{\prime}},{ }^{y} \lambda_{t}^{s, s^{\prime}}$ and ${ }^{z} \lambda_{t}^{s, s^{\prime}}$ represent Lagrange multipliers.

$$
\begin{aligned}
& \left(P 2_{R L R}\right) \quad \phi_{R L R}\left({ }^{b} \lambda,{ }^{x} \lambda,{ }^{y} \lambda,{ }^{z} \lambda\right)= \\
& \min \sum_{s \in \mathcal{S}} p^{s} \sum_{t \in \mathcal{T}}\left({ }^{w} c_{t}^{s} w_{t}^{s}+{ }^{x} c_{t}^{s} x_{t}^{s}+{ }^{y} c_{t}^{s} y_{t}^{s}+\sum_{i \in \mathcal{I}}{ }^{b} c_{i, t}^{s} b_{i, t}^{s}\right)+\sum_{\substack{s, s^{\prime} \in \mathcal{S} \\
s<s^{\prime}}}\left[\sum_{i \in \mathcal{I}}{ }^{b} \lambda_{i, 0}^{s, s^{\prime}}\left(b_{i, 1}^{s}-b_{i, 1}^{s^{\prime}}\right)+{ }^{y} \lambda_{0}^{s, s^{\prime}}\left(y_{1}^{s}-y_{1}^{s^{\prime}}\right)\right] \\
& \quad+\sum_{\left(s, s^{\prime}\right) \in \mathcal{L}^{0}} \sum_{t=1}^{\mathbf{t}\left(s, s^{\prime}\right)}\left(\sum_{i \in \mathcal{I}}{ }^{b} \lambda_{i, t}^{s, s^{\prime}}\left(b_{i, t+1}^{s}-b_{i, t+1}^{s^{\prime}}\right)+{ }^{y} \lambda_{t}^{s, s^{\prime}}\left(y_{t+1}^{s}-y_{t+1}^{s^{\prime}}\right)+{ }^{x} \lambda_{t}^{s, s^{\prime}}\left(x_{t}^{s}-x_{t}^{s^{\prime}}\right)\right) \\
& \quad+\sum_{\left(s, s^{\prime}\right) \in \mathcal{L}_{T}^{1}} \sum_{t \in \mathcal{T}}{ }^{z} \lambda_{t}^{s, s^{\prime}}\left(z_{t}^{s, s^{\prime}}-z_{t}^{s^{\prime}, s}\right)
\end{aligned}
$$

s.t. $\quad(3)$

$$
z_{t}^{s, s^{\prime}} \leq 1-b_{i, \tau}^{s} \quad \forall\left(s, s^{\prime}\right) \in \mathcal{L}_{T}^{1}, t \in \mathcal{T},\{i\}=\mathcal{D}\left(s, s^{\prime}\right), \tau \in \mathcal{T}, \tau \leq t
$$




$$
\begin{array}{ll}
z_{t}^{s, s^{\prime}} \geq 1-\sum_{\substack{\tau \in \mathcal{T} \\
\tau \leq t}} b_{i, \tau}^{s} & \forall\left(s, s^{\prime}\right) \in \mathcal{L}_{T}^{1}, t \in \mathcal{T},\{i\}=\mathcal{D}\left(s, s^{\prime}\right) \\
z_{t}^{s^{\prime}, s} \leq 1-b_{i, \tau}^{s^{\prime}} & \forall\left(s, s^{\prime}\right) \in \mathcal{L}_{T}^{1}, t \in \mathcal{T},\{i\}=\mathcal{D}\left(s, s^{\prime}\right), \tau \in \mathcal{T}, \tau \leq t \\
z_{t}^{s^{\prime}, s} \geq 1-\sum_{\substack{\tau \in \mathcal{T} \\
\tau \leq t}} b_{i, \tau}^{s^{\prime}} & \forall\left(s, s^{\prime}\right) \in \mathcal{L}_{T}^{1}, t \in \mathcal{T},\{i\}=\mathcal{D}\left(s, s^{\prime}\right) \\
w_{t}^{s} \in \mathcal{W}_{t}^{s}, x_{t}^{s} \in \mathcal{X}_{t}^{s}, y_{t}^{s} \in \mathcal{Y}_{t}^{s}, b_{i, t}^{s} \in\{0,1\} & \forall s \in \mathcal{S}, t \in \mathcal{T}, i \in \mathcal{I} \\
0 \leq z_{t}^{s, s^{\prime}}, z_{t}^{s^{\prime}, s} \leq 1 & \forall\left(s, s^{\prime}\right) \in \mathcal{L}_{T}^{1}, t \in \mathcal{T}
\end{array}
$$

Boolean variables $Z_{t}^{s, s^{\prime}}$ have been replaced by continuous variables $z_{t}^{s, s^{\prime}}$. As explained in section 6, variables $z_{t}^{s, s^{\prime}}$ will take discrete values even if the integrality condition is not imposed. Constraints (21a)-(21b) and (22a)-(22b) are linear algebraic formulations of (18) and (19) respectively. $P 2_{R L R}$ is therefore an MILP model and clearly a relaxation of $P 2_{\text {ref }}$ (and hence of $P 2$ ) for any values of the Lagrange multipliers. Thus,

$$
\phi_{R L R}\left({ }^{b} \lambda,{ }^{x} \lambda,{ }^{y} \lambda,{ }^{z} \lambda\right) \leq \phi \quad \forall\left({ }^{b} \lambda,{ }^{x} \lambda,{ }^{y} \lambda,{ }^{z} \lambda\right)
$$

Further, $P 2_{R L R}$ can be decomposed into one MILP sub-problem for each scenario. Note that since the disjunctions have been completely relaxed, $P 2_{R L R}$ is not the Lagrangean relaxation of $P 2_{\text {ref }}$. Therefore, we refer to it as the "relaxed" Lagrangean relaxation of $P 2_{\text {ref }}$. Then, the relaxed Lagrangean dual problem (Guignard and Kim (1987), Nemhauser and Wolsey (1988)) corresponding to $P 2_{\text {ref }}$ is

$$
\phi_{R L D}=\max _{{ }^{b} \lambda,{ }^{x} \lambda,{ }_{\lambda} \lambda, z \lambda} \phi_{R L R}\left({ }^{b} \lambda,{ }^{x} \lambda,{ }^{y} \lambda,{ }^{z} \lambda\right)
$$

Clearly, $\phi_{R L D}$ gives a lower bound to $\phi$. The solution of the Lagrangean dual may not satisfy the relaxed disjunctions. The penalty term corresponding to (20) tries to force $Z_{t}^{s, s^{\prime}}=Z_{t}^{s^{\prime}, s}$ and hence $\left[\bigwedge_{\tau=1}^{t}\left(\neg b_{i, \tau}^{s}\right)\right]=\left[\bigwedge_{\tau=1}^{t}\left(\neg b_{i, \tau}^{s^{\prime}}\right)\right]$ for all $\left(s, s^{\prime}\right) \in \mathcal{L}_{T}^{1}, t \in \mathcal{T},\{i\}=\mathcal{D}\left(s, s^{\prime}\right)$. Since $\left(s, s^{\prime}\right) \in \mathcal{L}_{T}^{1}$, therefore scenarios $s, s^{\prime}$ differ only in realization for $\theta_{i}$. Thus forcing decisions for source $i$ to follow non-anticipativity should force other decisions to follow non-anticipativity too. This motivates the inclusion of (19) and (20) in model $P 2_{\text {ref }}$.

Lower bounds at each node are generated by solving one such Lagrangean dual problem. Model $P$ represents the problem at any node in the branch and bound tree.

(P) $\phi_{P}=\min \sum_{s \in \mathcal{S}} p^{s} \sum_{t \in \mathcal{T}}\left({ }^{w} c_{t}^{s} w_{t}^{s}+{ }^{x} c_{t}^{s} x_{t}^{s}+{ }^{y} c_{t}^{s} y_{t}^{s}+\sum_{i \in \mathcal{I}}{ }^{b} c_{i, t}^{s} b_{i, t}^{s}\right)$

s.t. $(3),(18),(19)$ 


$$
\begin{aligned}
& \left.\begin{array}{lll}
x_{t}^{s} & =x_{t}^{s^{\prime}} & \text { if } t>0 \\
b_{i, t+1}^{s}=b_{i, t+1}^{s^{\prime}} & \forall i \in \mathcal{I} & \text { if } t \leq T-1 \\
y_{t+1}^{s}=y_{t+1}^{s^{\prime}} & \text { if } t \leq T-1
\end{array}\right\} \quad \forall\left(s, s^{\prime}, t\right) \in \mathcal{N}_{P}^{e} \\
& {\left[\begin{array}{llll}
Z_{t}^{s, s^{\prime}} & & \\
x_{t}^{s} & = & x_{t}^{s^{\prime}} & \\
b_{i, t+1}^{s} & = & b_{i, t+1}^{s^{\prime}} \\
y_{t+1}^{s} & = & y_{t+1}^{s^{\prime}} & \text { if } t \leq T-1
\end{array}\right] \vee\left[\neg Z_{t}^{s, s^{\prime}}\right] \forall\left(s, s^{\prime}, t\right) \in \mathcal{N}_{P}^{d}} \\
& Z_{t}^{s, s^{\prime}}=Z_{t}^{s^{\prime}, s} \quad \forall\left(s, s^{\prime}, t\right) \in \mathcal{N}_{P}^{d} \\
& w_{t}^{s} \in \mathcal{W}_{t}^{s}, x_{t}^{s} \in \mathcal{X}_{t, P}^{s}, y_{t}^{s} \in \mathcal{Y}_{t, P}^{s}, b_{i, t}^{s} \in \mathcal{B}_{i, t, P}^{s} \quad \forall s \in \mathcal{S}, t \in \mathcal{T}, i \in \mathcal{I} \\
& Z_{t}^{s, s^{\prime}}, Z_{t}^{s^{\prime}, s} \in \mathcal{Z}_{t, P}^{s^{\prime}, s} \quad \forall\left(s, s^{\prime}\right) \in \mathcal{L}_{T}^{1}, t \in \mathcal{T}
\end{aligned}
$$

$\mathcal{N}_{P}^{e}$ represents the set of tuples $\left(s, s^{\prime}, t\right)$ for which equality non-anticipativity constraints link variables $x_{t}^{(\cdot)}, y_{t+1}^{(\cdot)}$ and $b_{i, t+1}^{(\cdot)}$ for scenarios $s, s^{\prime}$ in problem $P$. Similarly, $\mathcal{N}_{P}^{d}$ represents the set of tuples $\left(s, s^{\prime}, t\right)$ for which non-anticipativity constraints are applied in the form of disjunctions in problem $P$. For example, for model $P 2_{\text {ref }}$

$$
\begin{gathered}
\mathcal{N}_{P 2_{\text {ref }}}^{e}=\left\{\left(s, s^{\prime}, t\right) \mid\left(s, s^{\prime}\right) \in \mathcal{L}^{0}, t \in \mathcal{T}, t \leq \mathbf{t}\left(s, s^{\prime}\right)\right\} \cup\left\{\left(s, s^{\prime}, 0\right) \mid s, s^{\prime} \in \mathcal{S}, s<s^{\prime}\right\} . \\
\mathcal{N}_{P 2_{\text {ref }}}^{d}=\left\{\left(s, s^{\prime}, t\right) \mid\left(s, s^{\prime}\right) \in \mathcal{L}_{T}^{1}, t \in \mathcal{T}\right\}
\end{gathered}
$$

$\mathcal{X}_{t, P}^{s}, \mathcal{Y}_{t, P}^{s}, \mathcal{B}_{i, t, P}^{s}$ and $\mathcal{Z}_{t, P}^{s, s^{\prime}}$ represent the bounds and domain restrictions on variables $x_{t}^{s}, y_{t}^{s}, b_{i, t}^{s}$ and $Z_{t}^{s, s^{\prime}}$ respectively, in model $P$. The (relaxed) Lagrangean relaxation problem corresponding to $P$ is obtained by relaxing all disjunctions for $\left(s, s^{\prime}, t\right) \in \mathcal{N}_{P}^{d}$ and replacing the symmetry conditions on variables $Z_{t}^{s, s^{\prime}}$ with respect to $\left(s, s^{\prime}\right)$ and the equality constraints corresponding to $\left(s, s^{\prime}, t\right) \in \mathcal{N}_{P}^{e}$ by penalty terms in the objective function. The corresponding relaxed Lagrangean dual is solved to obtain the lower bound for problem $P$.

In general, the solution of the Lagrangean dual may not satisfy the relaxed disjunctions and the dualized equality constraints. Feasible solutions can be generated at each node by applying problemspecific heuristics to the solution of the Lagrangean dual.

The branching step partitions the feasible space by branching on violated equality constraints and disjunctions. The strategy used for branching on dualized equality constraints is similar to that used by Caroe and Schultz (1999). Branching on equality constraints linking variables $b_{i, t}^{(\cdot)}$, or the binary components of variables $x_{t}^{(\cdot)}$ or $y_{t}^{(\cdot)}$, across scenarios $s, s^{\prime}$ is based on the standard dichotomy branching strategy. When branching on constraint $x_{l, t}^{s}=x_{l, t}^{s^{\prime}}$, where $x_{l, t}^{(\cdot)}$ is a continuous component of $x_{t}^{(\cdot)}$, the feasible space is partitioned about $\tilde{x}_{l, t}^{s, s^{\prime}}=\left(p^{s} \hat{x}_{l, t}^{s}+p^{s^{\prime}} \hat{x}_{l, t}^{s^{\prime}}\right) /\left(p^{s}+p^{s^{\prime}}\right)$, which is the mean value of variables $x_{l, t}^{s}$ and $x_{l, t}^{s^{\prime}}$ in the solution of the Lagrangean dual. The same strategy is used for branching on equality constraints on continuous components of variables $y_{t}^{(\cdot)}$. 
When branching on a relaxed disjunction corresponding to $\left(s, s^{\prime}, t\right) \in \mathcal{N}_{P}^{d}$, the feasible region is bifurcated into regions where $Z_{t}^{s, s^{\prime}}=Z_{t}^{s^{\prime}, s}=$ True and $Z_{t}^{s, s^{\prime}}=Z_{t}^{s^{\prime}, s}=$ False, respectively. The set of dualized equality constraints on the up-branch $\left(Z_{t}^{s, s^{\prime}}=Z_{t}^{s^{\prime}, s}=\right.$ True $)$ is augmented by the set of equality constraints inside the disjunction corresponding to $\left(s, s^{\prime}, t\right)$. Note that the solution of the relaxed Lagrangean dual of problem $P$ may be such that $\hat{Z}_{t}^{s, s^{\prime}}=\hat{Z}_{t}^{s^{\prime}, s}=$ True. Thus, introducing the restriction $Z_{t}^{s, s^{\prime}}=Z_{t}^{s^{\prime}, s}=$ True may not alter the solution of the Lagrangean dual. Thus, the first branch is further bifurcated to eliminate infeasibility in one of the violated equality constraints (see branching sub-step in Fig. 2).

The problem at hand will govern the order in which the dualized equality constraints and the relaxed disjunctions are chosen for branching. Although we do not mention this step in Fig. 2, logic inferencing on Boolean and discrete variables can significantly impact the quality of the lower bounds. In this algorithm, constraints (4a), (18) and (19) can be used for logic inferencing on variables $b$ and $Z$. For example, consider problem $P$ with bound $b_{i_{1}, 1}^{s_{1}} \leq 0$. We can use (18) to infer that $Z_{1}^{s_{1}, s_{2}}=$ True for $s_{2}$ such that $\mathcal{D}\left(s_{1}, s_{2}\right)=\left\{i_{1}\right\}$. The set of equality constraints (to be dualized) can therefore be augmented as $\mathcal{N}_{P}^{e}:=\mathcal{N}_{P}^{e} \cup\left(s_{1}, s_{2}, 1\right)$. Also, we can use (4a) to infer that $b_{i_{1}, 1}^{s} \leq 0 \forall s \in \mathcal{S}$. These changes to problem $P$ may impact the value of the Lagrangean dual significantly. Therefore, it is important to use the logic inferencing step before the Lagrangean dual is solved in step 3 of the algorithm.

It should be noted that if some components of variables $x_{t}$ and $y_{t}$ are continuous, then some stopping criterion is needed to avoid infinite branching on these components. As explained by Caroe and Schultz (1999), if the feasible region is bounded and if we branch parallel to the coordinate axes, then we can stop after the $l_{\infty}$-diameter of the feasible sets of the sub-problems has fallen below a certain threshold. The algorithm is then guaranteed to converge finitely.

\section{Numerical results}

In Example 1 we illustrate the advantage of our modeling approach compared to the expected value solution approach. We also show the effect of the properties presented in section 7 on the size of the MILP reformulation of the model. Example 2 presents computational results for the proposed branch and bound algorithm. All problems are solved using ILOG CPLEX 9.0 on a Pentium-IV, 2.4 GHz Linux machine.

Example 1. Fig. 3 shows a process network that can be used to produce chemical A. The demand for $\mathrm{A}$ has to be met in each time period over a ten time period time-horizon. Currently, A is being produced in unit 3 from chemical $\mathrm{B}$, which is purchased from the market. Units 1 and 2, which are based on new technology can produce $\mathrm{B}$ from raw materials $\mathrm{C}$ and $\mathrm{D}$, respectively, which can be 


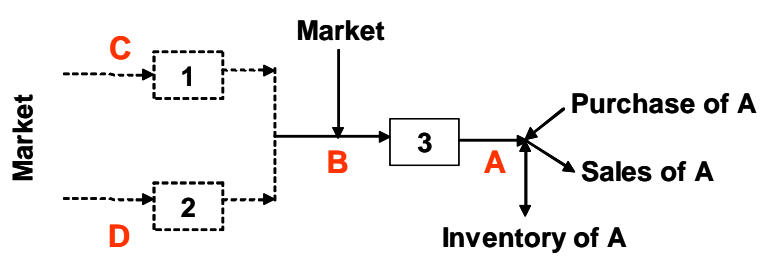

Figure 3: Example 1

purchased from the market. If needed, A can also be purchased from the market. Also, inventory of chemical A can be maintained.

Decisions to be made in each time period include selection of units to be installed or expanded (variables $b$ ), capacities of units and feed flow rates (variables $y$ ), and purchase and sales to satisfy demand of A (variables $x$ ).

The yields (tons of product per ton of raw material) of units 1 and 2 are uncertain. The uncertainty in yield will be resolved only after the unit has been installed and operated for one time period. Thus the optimization decisions will determine when this uncertainty is resolved. Hence the yield uncertainty is endogenous.

In general, uncertainty in demand of chemical A can be the source of exogenous uncertainty. However, for ease of illustration we do not consider exogenous uncertainty. Unit 3 is already operational with an existing capacity of 3 tons/hour and known yield of 0.70 . Possible realizations for yield of unit 1 are 0.71 and 0.79 , both possibilities being equally likely. Similarly, 0.65 and 0.85 are equally probable realizations for yield of unit 2 . Note that the mean yield for both these units is 0.75 . However, the yield for unit 2 has greater variance.

For sake of brevity, we do not present the detailed formulation and the data for this example. Interested readers may contact the authors. We compare the solution of the stochastic program $P 2$ with that obtained from the deterministic approach where the expected value problem is solved and the solution implemented partially till some uncertainty is resolved. The deterministic model is then updated and re-solved to obtain optimal decisions for the future. The solution of the expected value problem proposes installation of unit 1 and expansion of unit 3 in time period 1 (Fig. 4(a)). The approach proposes no more investments irrespective of the realization of yield for unit 1 . The expected cost for this solution is US $\$ 422,868$.

The solution of $P 2$ proposes the expansion of unit 3 and the installation of unit 2 with a small capacity in time period 1 (Fig. 4(b)). Proposed investments in time period 2 are based on the realization of yield of unit 2 . If yield of unit 2 is found to be 0.85 , that unit should be expanded further in time period 2. Otherwise, unit 1 should be installed in time period 2. The expected cost for this solution is US $\$ 409,222$. Therefore, the value of stochastic solution for this problem is US 


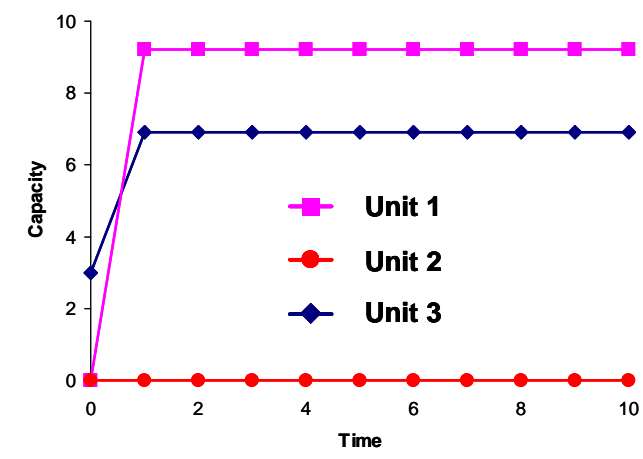

(a) Deterministic approach

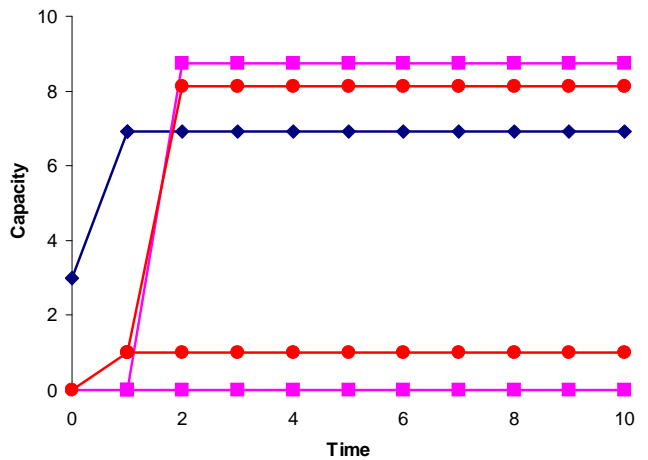

(b) Stochastic programming approach

Figure 4: Solutions for Example 1

\begin{tabular}{|l|rrr|}
\hline & $P 1$ & $P 2$ & \% Reduction \\
\hline Binary variables & 240 & 240 & 0.00 \\
Continuous variables & 985 & 965 & 2.03 \\
Constraints & 3,853 & 2,601 & 32.49 \\
CPU seconds & 92 & 69 & 25.00 \\
\hline
\end{tabular}

Table 1: Effect of Theorems 1 and 2 on size of MILP model for Example 1

$\$ 13,646$.

The stochastic program realizes the "value of information" associated with the yield of unit 2 . Therefore, the solution proposes small investment to resolve the uncertainty in unit 2 and appropriate recourse is proposed based on the realization of yield for this unit. The reduction in expected cost in excess of $3.2 \%$ is a result of this flexibility provided by the stochastic program.

Table 1 compares the big-M reformulations of models $P 1$ and $P 2$ in terms of their sizes. The CPU time corresponds to the time required for these models to be solved using ILOG CPLEX 9.0. As can be seen, Theorems 1 and 2 presented in section 7 lead to $32.49 \%$ reduction in the number of constraints and hence $25 \%$ reduction in the solution time.

Example 2. We compare the performance of the proposed algorithm with the standard LP based branch and bound algorithm applied to big-M formulations of four instances of the sizes problem (3). The constraints represented by (3) for the sizes problem are presented in appendix D. The model specifications for these problems are presented in Table 2, where $I, T$ and $S$ represent the number of sizes, time periods and scenarios, respectively. Note that we only consider endogenous uncertainty in problems A-D. Also, because the demands in each time period are fairly high $(\approx 10,000)$, variables $y_{i, t}^{s}$ (number of units produced) and $x_{i, i^{\prime}, t}^{s}$ (number of units substituted) are not restricted 


\begin{tabular}{|c|cccccc|}
\hline \multicolumn{7}{|c|}{ Problem } \\
\hline Name & $I$ & $T$ & $S$ & $\begin{array}{c}\text { Binary } \\
\text { variables }\end{array}$ & $\begin{array}{c}\text { Continuous } \\
\text { variables }\end{array}$ & Constraints \\
\hline A & 5 & 5 & 16 & 400 & 4,977 & 13,927 \\
B & 4 & 5 & 27 & 540 & 6,103 & 22,160 \\
C & 6 & 6 & 32 & 1,152 & 18,081 & 52,581 \\
D & 7 & 7 & 64 & 3,136 & 60,993 & 181,939 \\
\hline
\end{tabular}

Table 2: Model specifications for sample problems in Example 2

\begin{tabular}{|c|c|c|c|c|c|c|c|c|c|c|}
\hline \multirow{5}{*}{$\begin{array}{l}\text { Problem } \\
\text { Name }\end{array}$} & \multirow{2}{*}{\multicolumn{4}{|c|}{$\begin{array}{c}\text { Proposed Branch and Bound } \\
\text { After } t \text { CPU seconds }\end{array}$}} & \multicolumn{6}{|c|}{ LP based Branch and Bound } \\
\hline & & & & & \multicolumn{3}{|c|}{ After $2 \cdot t$ CPU seconds } & \multicolumn{3}{|c|}{ After $10 \cdot t$ CPU seconds } \\
\hline & \multirow{3}{*}{$t$} & \multirow{3}{*}{ Nodes } & \multicolumn{2}{|l|}{ Best } & \multicolumn{3}{|c|}{ Best } & \multicolumn{3}{|c|}{ Best } \\
\hline & & & sol. & $\%$ & Nodes & sol. & $\%$ & Nodes & sol. & $\%$ \\
\hline & & & found & gap & & found & gap & & found & gap \\
\hline A & 502 & 9 & 120,026 & 0.010 & 3,061 & 120,044 & 0.111 & 26,490 & 120,026 & 0.025 \\
\hline B & 430 & 4 & 112,608 & 0.010 & 708 & 112,623 & 0.258 & 6,899 & 112,621 & 0.157 \\
\hline $\mathrm{C}$ & 11,546 & 15 & 144,054 & 0.065 & 10,963 & 144,169 & 0.411 & 79,844 & 144,154 & 0.332 \\
\hline $\mathrm{D}$ & 13,507 & 3 & 245,930 & 0.038 & 15,591 & 246,029 & 0.247 & 71,151 & 246,026 & 0.237 \\
\hline
\end{tabular}

Table 3: Computational results for Example 2

to have integer values.

Table 3 compares the status of the proposed algorithm after $t$ seconds with that of the standard LP based branch and bound algorithm (ILOG CPLEX 9.0) after $2 \cdot t$ and $10 \cdot t$ CPU seconds. Clearly, the LP based branch and bound is not able to obtain the same optimality gap even after one order of magnitude more CPU time. Also, in comparable CPU time the proposed branch and bound algorithm generates better feasible solutions. This can be attributed in part to the tighter lower bounds obtained from the relaxed Lagrangean dual. Table 4 compares the lower bounds generated by the two algorithms at the root node of the branch and bound tree. The gaps are calculated relative to the best solution found (column 4 of Table 3).

We developed an object-oriented implementation for the proposed branch and bound algorithm in $\mathrm{C}++$. In our implementation, the Lagrangean dual problem at each node is solved using a

\begin{tabular}{|c|c|c|c|c|}
\hline \multirow{2}{*}{$\begin{array}{c}\text { Problem } \\
\text { Name }\end{array}$} & \multicolumn{2}{|c|}{ Proposed algorithm } & \multicolumn{2}{c|}{ ILOG CPLEX } \\
\cline { 2 - 5 } & $\begin{array}{c}\text { Lower bound } \\
\text { at root node }\end{array}$ & $\begin{array}{c}\% \\
\text { gap }\end{array}$ & $\begin{array}{c}\text { Lower bound } \\
\text { at root node }\end{array}$ & $\begin{array}{c}\% \\
\text { gap }\end{array}$ \\
\hline A & 120,003 & 0.019 & 119,770 & 0.213 \\
B & 112,595 & 0.012 & 112,240 & 0.327 \\
C & 143,742 & 0.217 & 143,466 & 0.408 \\
D & 245,403 & 0.214 & 245,341 & 0.239 \\
\hline
\end{tabular}

Table 4: Comparison of lower bounds at root node in Example 2 
sub-gradient procedure (Fisher (1981)). This procedure is stopped if the bound does not improve for a pre-specified number of iterations or if a total iteration limit is reached. At the root node, the Lagrange multipliers are initialized to zero. At all other nodes, the Lagrange multipliers are initialized to the optimal values at the parent node.

To generate feasible solutions, we apply a heuristic to the solution of the Lagrangean dual to obtain values for binary variables such that all non-anticipativity constraints on these variables are satisfied. The resulting LP is solved to generate feasible values for the continuous variables. The next node to be solved in the branch and bound tree is chosen based on the best lower bound rule. The dualized equality constraint with the highest penalty is chosen for branching. If the corresponding penalty is less than a pre-specified value, the disjunction with the maximum violation is selected for branching.

\section{Conclusions}

In this paper, we have addressed a class of stochastic programs where the optimization decisions determine when uncertainty is resolved. This class of problems is especially relevant to real world applications where the decision-maker has the option of obtaining information pro-actively by making some investments. We have extended the stochastic programming modeling framework by incorporating the interaction between the optimization decisions and the information discovery process through the use of disjunctive programming. We have presented theoretical properties that lead to significant reduction in the size of the proposed model. We have also presented a Lagrangean duality based branch and bound algorithm to solve the model.

Results show that the inclusion of the option of getting information at the cost of investments leads to significant improvements in the quality of the solution. Results presented for the branch and bound algorithm show more than one order of magnitude reduction in solution time.

\section{Acknowledgments}

Financial support from the ExxonMobil Upstream Research Company is gratefully acknowledged. The authors are specially thankful to Amr El-Bakry and Eric Mulkay from ExxonMobil Upstream Research Company for providing guidance in the implementation of the branch and bound algorithm. 


\section{References}

Ahmed, S., 2000. Strategic planning under uncertainty: Stochastic integer programming approaches. Ph.D. thesis, University of Illinois at Urbana-Champaign.

Balas, E., 1985. Disjunctive programming and a hierarchy of relaxations for discrete optimization problems. Siam Journal on Algebraic and Discrete Methods 6 (3), 466-486.

Birge, J. R., 1997. Stochastic programming computation and applications. INFORMS J. Comput. 9, 111-133.

Caroe, C. C., Schultz, R., 1999. Dual decomposition in stochastic integer programming. Oper. Res. Lett. 24, 37-45.

Fisher, M. L., 1981. The lagrangian-relaxation method for solving integer programming-problems. Management Science 27 (1), 1-18.

Goel, V., Grossmann, I. E., 2004. A stochastic programming approach to planning of offshore gas field developments under uncertainty in reserves. Computers and Chemical Engineering 28 (8), 1409-1429.

Guignard, M., Kim, S., 1987. Lagrangean decomposition - a model yielding stronger lagrangean bounds. Mathematical Programming 39 (2), 215-228.

Held, H., Woodruff, D. L., 2003. Heuristics for multi-stage interdiction of stochastic networks. Journal of Heuristics (Submitted for publication).

Jonsbraten, T. W., 1998. Optimization models for petroleum field exploitation. Ph.D. thesis, Norwegian School of Economics and Business Administration.

Jonsbraten, T. W., Wets, R. J. B., Woodruff, D. L., 1998. A class of stochastic programs with decision dependent random elements. Annals of Operations Research 82, 83-106.

Jorjani, S., Scott, C. H., Woodruff, D. L., 1999. Selection of an optimal subset of sizes. International Journal of Production Research 37 (16), 3697-3710.

Krarup, J., Bilde, O., 1977. Plant location, set covering and economic lot size: an o(mn)-algorithm for structured problems. In: Collatz, L., Wetterling, W. (Eds.), Optimierung bei graphentheoretischen and ganzzahligen Problemen. Vol. 36 of International Series of Numerical Mathematics. Birkhauser Verlag, Basel, pp. 155-180.

Nemhauser, G. L., Wolsey, L. A., 1988. Integer and combinatorial optimization. Wiley, New York, english Series: Wiley-Interscience series in discrete mathematics and optimization. 
Pflug, G., 1990. On-line optimization of simulated markovian processes. Math. Oper. Res. 15, No.3, 381-395.

Ruszczynski, A., 1997. Decomposition methods in stochastic programming. Math. Programming (Ser. B) 79, 333-353.

Sahinidis, N. V., 2004. Optimization under uncertainty: State-of-the-art and opportunities. Comput. Chem. Eng. 28 (6-7), 971-983.

Schultz, R., 2003. Stochastic programming with integer variables. Mathematical Programming 97 (1-2), 285-309.

Turkay, M., Grossmann, I. E., 1996. Disjunctive programming techniques for the optimization of process systems with discontinuous investment costs multiple size regions. Ind. Eng. Chem. Res. $35,2611-2623$.

Viswanath, K., Peeta, S., Salman, S., 2004. Investing in the links of a stochastic network to minimize expected shortest path length (Working paper).

\section{A Proof of Proposition 1}

Suppose vectors $\hat{b}^{s}, \hat{b}^{s^{\prime}}, \hat{Z}^{s, s^{\prime}}$ satisfy (9)-(11).

Proof of (a). Consider $t \in \mathcal{T}, t \leq \hat{t}$. Let

$$
\begin{aligned}
& \bigwedge_{i \in \mathcal{D}\left(s, s^{\prime}\right)}\left[\bigwedge_{\tau=1}^{t}\left(\neg \hat{b}_{i, \tau}^{s}\right)\right]=\text { True. } \\
& \Rightarrow \bigwedge_{i \in \mathcal{D}\left(s, s^{\prime}\right)}\left[\bigwedge_{\tau=1}^{t^{\prime}}\left(\neg \hat{b}_{i, \tau}^{s}\right)\right]=\text { True } \forall t^{\prime} \in \mathcal{T}, t^{\prime} \leq t
\end{aligned}
$$

Since $t \leq \hat{t}$ and $\left(\hat{b}^{s}, \hat{b}^{s^{\prime}}, \hat{Z}^{s, s^{\prime}}\right)$ satisfies (11), therefore,

$$
\hat{Z}_{t^{\prime}}^{s, s^{\prime}}=\text { True } \forall t^{\prime} \in \mathcal{T}, t^{\prime} \leq t
$$

Using index $\tau$ instead of $t^{\prime}$,

$$
\hat{Z}_{\tau}^{s, s^{\prime}}=\text { True } \quad \forall \tau \in \mathcal{T}, \tau \leq t
$$

Using (10), we get

$$
\hat{b}_{i, \tau+1}^{s}=\hat{b}_{i, \tau+1}^{s^{\prime}} \quad \forall i \in \mathcal{I}, \tau \in \mathcal{T}, \tau \leq t
$$

Also, from (9),

$$
\hat{b}_{i, \tau}^{s}=\hat{b}_{i, \tau}^{s^{\prime}} \quad \forall i \in \mathcal{I}, \tau=1
$$


Combining, we get

$$
\hat{b}_{i, \tau}^{s}=\hat{b}_{i, \tau}^{s^{\prime}} \quad \forall i \in \mathcal{I}, \tau \in \mathcal{T}, \tau \leq t+1
$$

Thus,

$$
\bigwedge_{i \in \mathcal{D}\left(s, s^{\prime}\right)}\left[\bigwedge_{\tau=1}^{t}\left(\neg \hat{b}_{i, \tau}^{s}\right)\right] \Rightarrow\left[\hat{b}_{i, \tau}^{s}=\hat{b}_{i, \tau}^{s^{\prime}} \quad \forall i \in \mathcal{I}, \tau \in \mathcal{T}, \tau \leq t+1\right] .
$$

Proof of (b). Suppose

$$
\begin{gathered}
\bigwedge_{i \in \mathcal{D}\left(s, s^{\prime}\right)}\left[\bigwedge_{\tau=1}^{\hat{t}+1}\left(\neg \hat{b}_{i, \tau}^{s}\right)\right]=\text { True. } \\
\Rightarrow \bigwedge_{i \in \mathcal{D}\left(s, s^{\prime}\right)}\left[\bigwedge_{\tau=1}^{\hat{t}}\left(\neg \hat{b}_{i, \tau}^{s}\right)\right]=\text { True. }
\end{gathered}
$$

Now using the result of part (a), we get,

$$
\hat{b}_{i, \tau}^{s}=\hat{b}_{i, \tau}^{s^{\prime}} \quad \forall i \in \mathcal{I}, \tau \in \mathcal{T}, \tau \leq \hat{t}+1
$$

Thus, for $t=\hat{t}+1$,

$$
\bigwedge_{i \in \mathcal{D}\left(s, s^{\prime}\right)}\left[\bigwedge_{\tau=1}^{t}\left(\neg \hat{b}_{i, \tau}^{s}\right)\right] \Rightarrow\left[\hat{b}_{i, \tau}^{s}=\hat{b}_{i, \tau}^{s^{\prime}} \quad \forall i \in \mathcal{I}, \tau \in \mathcal{T}, \tau \leq t\right] .
$$

Proof of (c). Consider $t \in \mathcal{T}, t \leq \hat{t}+1$.

$(\Rightarrow)$ :

Suppose,

$$
\bigwedge_{i \in \mathcal{D}\left(s, s^{\prime}\right)}\left[\bigwedge_{\tau=1}^{t}\left(\neg \hat{b}_{i, \tau}^{s}\right)\right]=\text { True }
$$

If $t \leq \hat{t}$, then from (a), we get

$$
\hat{b}_{i, \tau}^{s}=\hat{b}_{i, \tau}^{s^{\prime}} \quad \forall i \in \mathcal{I}, \tau \in \mathcal{T}, \tau \leq t+1
$$

If $t=\hat{t}+1$, then from (b), we get

$$
\hat{b}_{i, \tau}^{s}=\hat{b}_{i, \tau}^{s^{\prime}} \quad \forall i \in \mathcal{I}, \tau \in \mathcal{T}, \tau \leq t
$$

In either case, we have

$$
\hat{b}_{i, \tau}^{s}=\hat{b}_{i, \tau}^{s^{\prime}} \quad \forall i \in \mathcal{I}, \tau \in \mathcal{T}, \tau \leq t
$$


Therefore,

$$
\bigwedge_{i \in \mathcal{D}\left(s, s^{\prime}\right)}\left[\bigwedge_{\tau=1}^{t}\left(\neg \hat{b}_{i, \tau}^{s^{\prime}}\right)\right]=\text { True }
$$

Thus,

$$
\bigwedge_{i \in \mathcal{D}\left(s, s^{\prime}\right)}\left[\bigwedge_{\tau=1}^{t}\left(\neg \hat{b}_{i, \tau}^{s}\right)\right] \Rightarrow \bigwedge_{i \in \mathcal{D}\left(s, s^{\prime}\right)}\left[\bigwedge_{\tau=1}^{t}\left(\neg \hat{b}_{i, \tau}^{s^{\prime}}\right)\right] \quad \forall t \in \mathcal{T}, t \leq \hat{t}+1
$$

$(\Leftarrow)$ :

We prove the contra-positive of the converse. Suppose

$$
\bigwedge_{i \in \mathcal{D}\left(s, s^{\prime}\right)}\left[\bigwedge_{\tau=1}^{t}\left(\neg \hat{b}_{i, \tau}^{s}\right)\right]=\text { False }
$$

Thus, there exists $(i, \tau)$ such that $i \in \mathcal{D}\left(s, s^{\prime}\right), \tau \in \mathcal{T}, \tau \leq t$ for which $\hat{b}_{i, \tau}^{s}=1$. Define

$$
\tau^{*}=\min _{\tau}\left\{\tau \mid \tau \in \mathcal{T}, \tau \leq t \text { such that } \exists i \in \mathcal{D}\left(s, s^{\prime}\right), \hat{b}_{i, \tau}^{s}=1\right\}
$$

and

$$
i^{*} \in\left\{i \mid i \in \mathcal{D}\left(s, s^{\prime}\right), \hat{b}_{i, \tau^{*}}^{s}=1\right\}
$$

Case 1: $\tau^{*}=1$. By definition of $i^{*}$ and $\tau^{*}, \hat{b}_{i^{*}, \tau^{*}}^{s}=1$. Thus, using (9) we get

$$
\hat{b}_{i^{*}, \tau^{*}}^{s}=\hat{b}_{i^{*}, \tau^{*}}^{s^{\prime}}=1
$$

Since $\tau^{*}=1 \leq t$ and $i^{*} \in \mathcal{D}\left(s, s^{\prime}\right)$, therefore

$$
\bigwedge_{i \in \mathcal{D}\left(s, s^{\prime}\right)}\left[\bigwedge_{\tau=1}^{t}\left(\neg \hat{b}_{i, \tau}^{s^{\prime}}\right)\right]=\text { False }
$$

Case 2: $\tau^{*}>1$ : By definition of $\tau^{*}, \hat{b}_{i, \tau}^{s}=0 \quad \forall i \in \mathcal{D}\left(s, s^{\prime}\right), \tau \in \mathcal{T}, \tau \leq \tau^{*}-1$. Thus,

$$
\bigwedge_{i \in \mathcal{D}\left(s, s^{\prime}\right)}\left[\bigwedge_{\tau=1}^{\tau^{*}-1}\left(\neg \hat{b}_{i, \tau}^{s}\right)\right]=\text { True }
$$

Now, since $1<\tau^{*} \leq t \leq \hat{t}+1$ and $\tau^{*} \in \mathcal{T}=\{1,2, \ldots, T\}$, therefore $2 \leq \tau^{*} \leq \hat{t}+1$. Thus, $\tau^{*}-1 \in \mathcal{T}$ and $1 \leq \tau^{*}-1 \leq \hat{t}$. Then, using the result of part (a) together with (A.3), we get

$$
\hat{b}_{i, \tau}^{s}=\hat{b}_{i, \tau}^{s^{\prime}} \quad \forall i \in \mathcal{I}, \tau \in \mathcal{T}, \tau \leq \tau^{*}
$$


From definition of $\left(i^{*}, \tau^{*}\right), \hat{b}_{i^{*}, \tau^{*}}^{s}=1$. Using (A.4) we get $\hat{b}_{i^{*}, \tau^{*}}^{s^{\prime}}=1$. Now, since $\tau^{*} \leq t$ and $i^{*} \in \mathcal{D}\left(s, s^{\prime}\right)$, therefore,

$$
\bigwedge_{i \in \mathcal{D}\left(s, s^{\prime}\right)}\left[\bigwedge_{\tau=1}^{t}\left(\neg \hat{b}_{i, \tau}^{s^{\prime}}\right)\right]=\text { False }
$$

Combining results for (A.2) and (A.5), we get

$$
\neg\left(\bigwedge_{i \in \mathcal{D}\left(s, s^{\prime}\right)}\left[\bigwedge_{\tau=1}^{t}\left(\neg \hat{b}_{i, \tau}^{s}\right)\right]\right) \Rightarrow \neg\left(\bigwedge_{i \in \mathcal{D}\left(s, s^{\prime}\right)}\left[\bigwedge_{\tau=1}^{t}\left(\neg \hat{b}_{i, \tau}^{s^{\prime}}\right)\right]\right) \quad \forall t \in \mathcal{T}, t \leq \hat{t}+1
$$

Thus, from (A.1) and (A.6) we get

$$
\bigwedge_{i \in \mathcal{D}\left(s, s^{\prime}\right)}\left[\bigwedge_{\tau=1}^{t}\left(\neg \hat{b}_{i, \tau}^{s}\right)\right] \Leftrightarrow \bigwedge_{i \in \mathcal{D}\left(s, s^{\prime}\right)}\left[\bigwedge_{\tau=1}^{t}\left(\neg \hat{b}_{i, \tau}^{s^{\prime}}\right)\right] \quad \forall t \in \mathcal{T}, t \leq \hat{t}+1
$$

Proofs for (d) and (e). Follow directly by combining (c) with (a) and (b) respectively.

\section{B Lemma 1}

Lemma 1. Consider scenarios $s_{0}, s_{1}, s_{2}, \ldots, s_{r} \in \mathcal{S}$ and time period $\hat{t} \in \mathcal{T}, \hat{t} \leq T-1$. Suppose vectors $\hat{b}, \hat{Z}$ are such that for $k \in\{0,1, \ldots, r-1\}$,

(i) If $s_{k}<s_{k+1}$, then sub-vectors $\hat{b}^{s_{k}}, \hat{b}^{s_{k+1}}, \hat{Z}^{s_{k}, s_{k+1}}$ satisfy (9)-(11) for $\left(s, s^{\prime}\right)=\left(s_{k}, s_{k+1}\right)$

(ii) If $s_{k+1}<s_{k}$, then sub-vectors $\hat{b}^{s_{k+1}}, \hat{b}^{s_{k}}, \hat{Z}^{s_{k+1}, s_{k}}$ satisfy (9)-(11) for $\left(s, s^{\prime}\right)=\left(s_{k+1}, s_{k}\right)$.

Then, vector $\hat{b}$ satisfies

$$
\bigwedge_{k=0}^{r-1}\left[\bigwedge_{\tau=1}^{t}\left(\bigwedge_{i \in \mathcal{D}\left(s_{k}, s_{k+1}\right)}\left(\neg \hat{b}_{i, \tau}^{s_{k}}\right)\right)\right] \Leftrightarrow \bigwedge_{k=0}^{r-1}\left[\bigwedge_{\tau=1}^{t}\left(\bigwedge_{i \in \mathcal{D}\left(s_{k}, s_{k+1}\right)}\left(\neg \hat{b}_{i, \tau}^{s_{0}}\right)\right)\right] \quad \forall t \in \mathcal{T}, t \leq \hat{t}+1
$$

Proof. Consider $t \in \mathcal{T}$ such that $t \leq \hat{t}+1$. We will first show that

$$
\bigwedge_{\tau=1}^{t}\left(\bigwedge_{i \in \mathcal{D}\left(s_{k}, s_{k+1}\right)}\left(\neg \hat{b}_{i, \tau}^{s_{k}}\right)\right) \Rightarrow\left[\hat{b}_{i, \tau}^{s_{k}}=\hat{b}_{i, \tau}^{s_{k+1}} \quad \forall i \in \mathcal{I}, \tau \in \mathcal{T}, \tau \leq t\right] \quad k=0,1, \ldots, r-1
$$


(B.1) will be used repeatedly in the proof of the Lemma.

If $k \in\{0,1, \ldots, r-1\}$ and $s_{k}<s_{k+1}$, then from condition (i) sub-vectors $\hat{b}^{s_{k}}, \hat{b}^{s_{k+1}}, \hat{Z}^{s_{k}, s_{k+1}}$ satisfy (9)-(11) for $\left(s, s^{\prime}\right)=\left(s_{1}, s_{2}\right)$. Hence, we can combine statements (a) and (b) of Proposition 1 to get (B.1). On the other hand, if $s_{k+1}<s_{k}$ then from condition (ii), sub-vectors $\hat{b}^{s_{k+1}}, \hat{b}^{s_{k}}, \hat{Z}^{s_{k+1}, s_{k}}$ satisfy (9)-(11) for $\left(s, s^{\prime}\right)=\left(s_{k+1}, s_{k}\right)$. Hence, in this case we can combine statements (d) and (e) of Proposition 1 to infer (B.1). Thus, (B.1) holds for $k \in\{0,1, \ldots, r-1\}$ irrespective of whether $s_{k}<s_{k+1}$ or $s_{k+1}<s_{k}$. Now we prove the Lemma under consideration.

$(\Rightarrow)$ : Suppose

$$
\begin{aligned}
\bigwedge_{k=0}^{r-1} & {\left[\bigwedge_{\tau=1}^{t}\left(\bigwedge_{i \in \mathcal{D}\left(s_{k}, s_{k+1}\right)}\left(\neg \hat{b}_{i, \tau}^{s_{k}}\right)\right)\right]=\text { True } } \\
\Rightarrow & \bigwedge_{\tau=1}^{t}\left(\bigwedge_{i \in \mathcal{D}\left(s_{k}, s_{k+1}\right)}\left(\neg \hat{b}_{i, \tau}^{s_{k}}\right)\right)=\text { True } \forall k \in\{0,1, \ldots, r-1\}
\end{aligned}
$$

Using (B.1), we get

$$
\hat{b}_{i, \tau}^{s_{k}}=\hat{b}_{i, \tau}^{s_{k+1}} \quad \forall i \in \mathcal{I}, \tau \in \mathcal{T}, \tau \leq t, k \in\{0,1, \ldots, r-1\}
$$

Thus,

$$
\hat{b}_{i, \tau}^{s_{0}}=\hat{b}_{i, \tau}^{s_{1}}=\hat{b}_{i, \tau}^{s_{2}}=\cdots=\hat{b}_{i, \tau}^{s_{r}-1}=\hat{b}_{i, \tau}^{s_{r}} \quad \forall i \in \mathcal{I}, \tau \in \mathcal{T}, \tau \leq t
$$

Using this in the hypothesis, we get

$$
\bigwedge_{k=0}^{r-1}\left[\bigwedge_{\tau=1}^{t}\left(\bigwedge_{i \in \mathcal{D}\left(s_{k}, s_{k+1}\right)}\left(\neg \hat{b}_{i, \tau}^{s_{0}}\right)\right)\right]=\text { True }
$$

$(\Leftarrow)$ : Suppose

$$
\begin{aligned}
& \bigwedge_{k=0}^{r-1}\left[\bigwedge_{\tau=1}^{t}\left(\bigwedge_{i \in \mathcal{D}\left(s_{k}, s_{k+1}\right)}\left(\neg \hat{b}_{i, \tau}^{s_{0}}\right)\right)\right]=\text { True } \\
& \Rightarrow \bigwedge_{\tau=1}^{t}\left(\bigwedge_{i \in \mathcal{D}\left(s_{k}, s_{k+1}\right)}\left(\neg \hat{b}_{i, \tau}^{s_{0}}\right)\right)=\text { True } \forall k \in\{0,1, \ldots, r-1\}
\end{aligned}
$$

Therefore, $\bigwedge_{\tau=1}^{t}\left(\bigwedge_{i \in \mathcal{D}\left(s_{0}, s_{1}\right)}\left(\neg \hat{b}_{i, \tau}^{s_{0}}\right)\right)=$ True. Hence, from (B.1) we get

$$
\hat{b}_{i, \tau}^{s_{0}}=\hat{b}_{i, \tau}^{s_{1}} \quad \forall i \in \mathcal{I}, \tau \in \mathcal{T}, \tau \leq t
$$

Replacing $\hat{b}_{i, \tau}^{s_{0}}$ by $\hat{b}_{i, \tau}^{s_{1}}$ in (B.2) we can infer that $\bigwedge_{\tau=1}^{t}\left(\bigwedge_{i \in \mathcal{D}\left(s_{1}, s_{2}\right)}\left(\neg \hat{b}_{i, \tau}^{s_{1}}\right)\right)=$ True. Once again, using (B.1) we can infer that

$$
\hat{b}_{i, \tau}^{s_{1}}=\hat{b}_{i, \tau}^{s_{2}} \quad \forall i \in \mathcal{I}, \tau \in \mathcal{T}, \tau \leq t
$$


Hence, combining (B.2), (B.3) and (B.4) we can infer that $\bigwedge_{\tau=1}^{t}\left(\bigwedge_{i \in \mathcal{D}\left(s_{2}, s_{3}\right)}\left(\neg \hat{b}_{i, \tau}^{s_{2}}\right)\right)=$ True. We can continue the argument to show that,

$$
\hat{b}_{i, \tau}^{s_{0}}=\hat{b}_{i, \tau}^{s_{1}}=\hat{b}_{i, \tau}^{s_{2}}=\cdots=\hat{b}_{i, \tau}^{s_{r}} \quad \forall i \in \mathcal{I}, \tau \in \mathcal{T}, \tau \leq t
$$

Using this in the hypothesis we get

$$
\bigwedge_{k=0}^{r-1}\left[\bigwedge_{\tau=1}^{t}\left(\bigwedge_{i \in \mathcal{D}\left(s_{k}, s_{k+1}\right)}\left(\neg \hat{b}_{i, \tau}^{s_{k}}\right)\right)\right]=\text { True }
$$

\section{Proof of Proposition 2}

Proof. Suppose vectors $\hat{b}, \hat{x}, \hat{y}, \hat{Z}$ satisfy (i)-(iii) in the condition of the proposition. Consider scenarios $s_{a}, s_{b}$ such that

$$
\begin{array}{ll}
\theta_{i}^{s_{a}}=\theta_{i}^{s_{b}} & i \in\{1,2, \ldots, I-r\} \\
\theta_{i}^{s_{a}} \neq \theta_{i}^{s_{b}} & i \in\{I-r+1, I-r+2, \ldots, I\}
\end{array}
$$

Thus, $\mathcal{D}\left(s_{a}, s_{b}\right)=\{I-r+1, I-r+2, \ldots, I\}$ and $\left|\mathcal{D}\left(s_{a}, s_{b}\right)\right|=r$. We consider the case where $1 \leq r=\left|\mathcal{D}\left(s_{a}, s_{b}\right)\right| \leq I$. Without loss of generality, we assume that $s_{a}<s_{b}$ and that $s_{a}, s_{b}$ differ in realizations for the last last $r$ endogenous parameters.

Hence, $\left(s_{a}, s_{b}\right) \in \mathcal{L}^{1+}$. We will prove that vectors $\hat{b}, \hat{x}, \hat{y}, \hat{Z}$ satisfy constraints (15)-(16) for $\left(s_{a}, s_{b}, t\right)$ such that $t \in \mathcal{T}, t \leq \mathbf{t}\left(s_{a}, s_{b}\right)$. Since $\left(s_{a}, s_{b}\right) \in \mathcal{L}^{1+}$, this will establish that $(\hat{b}, \hat{x}, \hat{y}, \hat{Z})$ satisfies (15)-(16) for all $\left(s, s^{\prime}, t\right)$ such that $\left(s, s^{\prime}\right) \in \mathcal{L}^{1+}, t \in \mathcal{T}, t \leq \mathbf{t}\left(s, s^{\prime}\right)$. The result will follow.

Case 1: $\boldsymbol{t}\left(s_{a}, s_{b}\right)<T$. Scenarios $s_{a}, s_{b}$ differ in realizations of $r$ endogenous parameters. We construct $r$ "intermediate" scenarios indexed by variable indices $s_{1}, s_{2}, \ldots, s_{r}$. These scenarios are derived from scenario $s_{a}$ by progressively changing the realization of one of these $r$ endogenous parameters to the corresponding realization in scenario $s_{b}$. For example, scenario $s_{1}$ is identical to scenario $s_{a}$ except that $\theta_{I-r+1}^{s_{1}}=\theta_{I-r+1}^{s_{b}} \neq \theta_{I-r+1}^{s_{a}}$. Similarly, scenario $s_{2}$ is identical to scenario $s_{a}$ except that $\theta_{I-r+1}^{s_{2}}=\theta_{I-r+1}^{s_{b}} \neq \theta_{I-r+1}^{s_{a}}$ and $\theta_{I-r+2}^{s_{2}}=\theta_{I-r+2}^{s_{b}} \neq \theta_{I-r+2}^{s_{a}}$. Scenarios $s_{1}, s_{2}, \ldots, s_{r}$ are identical to scenario $s_{a}$ in terms of realizations for all exogenous parameters. Mathematically,

$$
\begin{aligned}
& \theta_{i}^{s_{1}}=\theta_{i}^{s_{a}} \quad \forall i \in \mathcal{I} \backslash\{I-r+1\}, \quad \theta_{i}^{s_{1}} \quad=\theta_{i}^{s_{b}} \quad \forall i \in\{I-r+1\} \\
& \theta_{i}^{s_{2}}=\theta_{i}^{s_{a}} \quad \forall i \in \mathcal{I} \backslash\{I-r+1, I-r+2\}, \quad \theta_{i}^{s_{2}} \quad=\theta_{i}^{s_{b}} \quad \forall i \in\{I-r+1, I-r+2\} \\
& \theta_{i}^{s_{r}}=\theta_{i}^{s_{a}} \quad \forall i \in \mathcal{I} \backslash\{I-r+1, \ldots, I\}, \quad \theta_{i}^{s_{r}} \quad=\theta_{i}^{s_{b}} \quad \forall i \in\{I-r+1, \ldots, I\}
\end{aligned}
$$


and

$$
\xi_{t}^{s_{k}}=\xi_{t}^{s_{a}} \quad \forall t \in \mathcal{T}, k \in\{1,2, \ldots, r\}
$$

Since we choose the set of scenarios as $\Xi \times\left(\times_{i \in \mathcal{I}} \Theta_{i}\right)$, therefore for any realization of the vector of exogenous parameters, $\xi=\left(\xi_{1}, \xi_{2}, \ldots, \xi_{T}\right)$, the set of scenarios includes scenarios corresponding to all possible combinations of realizations for the endogenous parameters. Therefore, the constructed scenarios corresponding to the indices $s_{1}, s_{2}, \ldots, s_{k}$ belong to the set of scenarios. Therefore, we can choose indices $s_{1}, s_{2}, \ldots, s_{k}$ such that $s_{1}, s_{2}, \ldots, s_{k} \in \mathcal{S}$.

By construction,

$$
\mathcal{D}\left(s_{a}, s_{1}\right)=\{I-r+1\}, \mathcal{D}\left(s_{1}, s_{2}\right)=\{I-r+2\}, \ldots, \mathcal{D}\left(s_{r-1}, s_{r}\right)=\{I\}
$$

and,

$$
\mathcal{D}\left(s_{r}, s_{b}\right)=\emptyset \text {. }
$$

Therefore,

$$
\left|\mathcal{D}\left(s_{a}, s_{1}\right)\right|=\left|\mathcal{D}\left(s_{1}, s_{2}\right)\right|=\ldots=\left|\mathcal{D}\left(s_{r-1}, s_{r}\right)\right|=1
$$

while

$$
\left|\mathcal{D}\left(s_{r}, s_{b}\right)\right|=0 .
$$

Also, by construction, $\xi_{t}^{s_{a}}=\xi_{t}^{s_{k}} \forall t \in \mathcal{T}, k \in\{1,2, \ldots, r\}$. Hence,

$$
\begin{gathered}
\mathbf{t}\left(s_{a}, s_{1}\right)=\mathbf{t}\left(s_{1}, s_{2}\right)=\cdots=\mathbf{t}\left(s_{r-1}, s_{r}\right)=T \text { and } \\
\mathbf{t}\left(s_{r}, s_{b}\right)=\mathbf{t}\left(s_{a}, s_{b}\right) .
\end{gathered}
$$

Consider scenarios $s_{a}$ and $s_{1}$.

Case 1a: $s_{a}<s_{1}$. Since $\left|\mathcal{D}\left(s_{a}, s_{1}\right)\right|=1$ and $\mathbf{t}\left(s_{a}, s_{1}\right)=T$, therefore $\left(s_{a}, s_{1}\right) \in \mathcal{L}_{T}^{1}$. Thus, from condition (iii) of the statement of this proposition, we can infer that vectors $\hat{b}, \hat{x}, \hat{y}, \hat{Z}$ ) satisfy (15)-(16) for $\left(s_{a}, s_{1}, t\right)$, where $t \in \mathcal{T}$. Since $\mathcal{D}\left(s_{a}, s_{1}\right)=\{I-r+1\}$, from (15) and (16) we have,

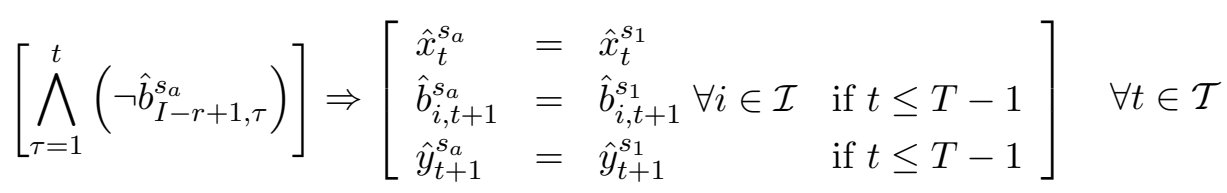

Case 1b: $s_{1}<s_{a}$. In this case, $\left(s_{1}, s_{a}\right) \in \mathcal{L}_{T}^{1}$. Therefore from condition (iii) of the statement of this proposition, we can infer that

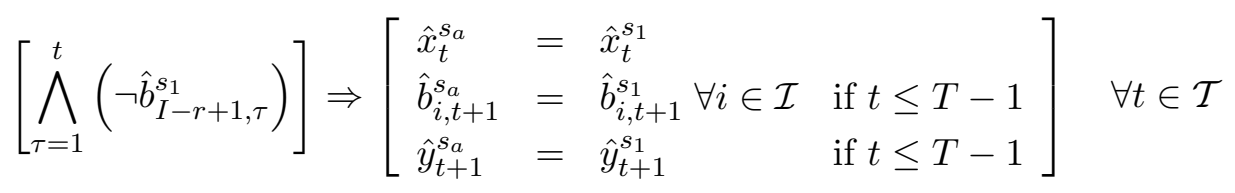


Also, since $s_{1}<s_{a}$, therefore vectors $\hat{b}, \hat{x}, \hat{y}, \hat{Z}$ satisfy (13a) for $\left(s_{1}, s_{a}, t=1\right)$. Thus, sub-vectors $\hat{b}^{s_{1}}, \hat{b}^{s_{a}}, \hat{Z}^{s_{1}, s_{a}}$ satisfy (13a) for $\left(s, s^{\prime}, t\right)=\left(s_{1}, s_{a}, 1\right)$ and $(15)-(16)$ for $\left(s, s^{\prime}\right)=\left(s_{1}, s_{a}\right), t \in \mathcal{T}$. Thus, sub-vectors $\hat{b}^{s_{1}}, \hat{b}^{s_{a}}, \hat{Z}^{s_{1}, s_{a}}$ satisfy (9)-(11) for $\left(s, s^{\prime}, \hat{t}\right)=\left(s_{1}, s_{a}, T-1\right)$. Thus, using $\mathcal{D}\left(s_{a}, s_{1}\right)=$ $\{I-r+1\}$ in statement (c) of Proposition 1, we get

$$
\left[\bigwedge_{\tau=1}^{t}\left(\neg \hat{b}_{I-r+1, \tau}^{s_{1}}\right)\right] \Leftrightarrow\left[\bigwedge_{\tau=1}^{t}\left(\neg \hat{b}_{I-r+1, \tau}^{s_{a}}\right)\right] \quad \forall t \in \mathcal{T}, t \leq \hat{t}+1=T
$$

Combining (C.1)-(C.3), we obtain that irrespective of whether $s_{a}<s_{1}$ or $s_{1}<s_{a}$,

$$
\left[\bigwedge_{\tau=1}^{t}\left(\neg \hat{b}_{I-r+1, \tau}^{s_{a}}\right)\right] \Rightarrow\left[\begin{array}{lll}
\hat{x}_{t}^{s_{a}} & =\hat{x}_{t}^{s_{1}} & \\
\hat{b}_{i, t+1}^{s_{a}}=\hat{b}_{i, t+1}^{s_{1}} & \forall i \in \mathcal{I} & \text { if } t \leq T-1 \\
\hat{y}_{t+1}^{s_{a}}=\hat{y}_{t+1}^{s_{1}} & \text { if } t \leq T-1
\end{array}\right] \quad \forall t \in \mathcal{T}
$$

Since

$$
\begin{gathered}
\left|\mathcal{D}\left(s_{1}, s_{2}\right)\right|=\left|\mathcal{D}\left(s_{2}, s_{3}\right)\right|=\cdots=\left|\mathcal{D}\left(s_{r-1}, s_{r}\right)\right|=1, \\
\mathbf{t}\left(s_{1}, s_{2}\right)=\mathbf{t}\left(s_{2}, s_{3}\right)=\cdots=\mathbf{t}\left(s_{r-1}, s_{r}\right)=T,
\end{gathered}
$$

and

$$
\mathcal{D}\left(s_{1}, s_{2}\right)=\{I-r+2\}, \mathcal{D}\left(s_{2}, s_{3}\right)=\{I-r+3\}, \ldots, \mathcal{D}\left(s_{r-1}, s_{r}\right)=\{I\},
$$

we can use the same logic as above to prove that

$$
\left.\begin{array}{rl}
{\left[\bigwedge_{\tau=1}^{t}\left(\neg \hat{b}_{I-r+2, \tau}^{s_{1}}\right)\right]} & \Rightarrow\left[\begin{array}{ll}
\hat{x}_{t}^{s_{1}}=\hat{x}_{t}^{s_{2}} \\
\hat{b}_{i, t+1}^{s_{1}}=\hat{b}_{i, t+1}^{s_{2}} \\
\hat{y}_{t+1}^{s_{1}}=\hat{y}_{t+1}^{s_{2}}
\end{array}\right] \quad \text { if } t \leq T-1
\end{array}\right] \quad \forall t \in \mathcal{T}
$$

Also, $\left|\mathcal{D}\left(s_{r}, s_{b}\right)\right|=0$. Therefore, depending on whether $s_{r}<s_{b}$ or $s_{b}<s_{r}$, either $\left(s_{r}, s_{b}\right) \in \mathcal{L}^{0}$ or $\left(s_{b}, s_{r}\right) \in \mathcal{L}^{0}$. In either case, from condition (ii) of this proposition we have

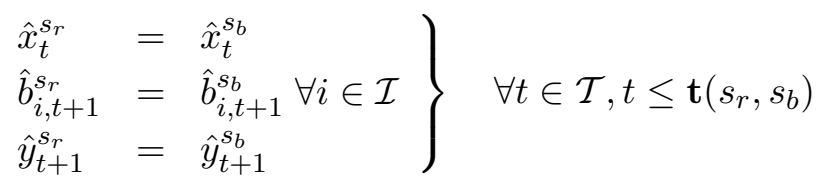

Now since $\mathbf{t}\left(s_{r}, s_{b}\right)=\mathbf{t}\left(s_{a}, s_{b}\right)$, therefore replacing $\mathbf{t}\left(s_{r}, s_{b}\right)$ by $\mathbf{t}\left(s_{a}, s_{b}\right)$ we get

$$
\left.\begin{array}{l}
\hat{x}_{t}^{s_{r}}=\hat{x}_{t}^{s_{b}} \\
\hat{b}_{i, t+1}^{s_{r}}=\hat{b}_{i, t+1}^{s_{b}} \forall i \in \mathcal{I} \\
\hat{y}_{t+1}^{s_{r}}=\hat{y}_{t+1}^{s_{b}}
\end{array}\right\} \quad \forall t \in \mathcal{T}, t \leq \mathbf{t}\left(s_{a}, s_{b}\right)
$$


Combining (C.4)-(C.7), we get

$$
\begin{aligned}
& \bigwedge_{\tau=1}^{t}\left[\left(\neg \hat{b}_{I-r+1, \tau}^{s_{a}}\right) \wedge\left(\neg \hat{b}_{I-r+2, \tau}^{s_{1}}\right) \wedge\left(\neg \hat{b}_{I-r+3, \tau}^{s_{2}}\right) \wedge \cdots \wedge\left(\neg \hat{b}_{I, \tau}^{s_{r-1}}\right)\right] \Rightarrow \\
& {\left[\begin{array}{llll}
\hat{x}_{t}^{s_{a}}=\hat{x}_{t}^{s_{1}}=\cdots=\hat{x}_{t}^{s_{r}}=\hat{x}_{t}^{s_{b}} \\
\hat{b}_{i, t+1}^{s_{a}}=\hat{b}_{i, t+1}^{s_{1}}=\cdots=\hat{b}_{i, t+1}^{s_{r}}=\hat{b}_{i, t+1}^{s_{b}} \forall i & \text { if } t \leq T-1 \\
\hat{y}_{t+1}^{s_{a}}=\hat{y}_{t+1}^{s_{1}}=\cdots=\hat{y}_{t+1}^{s_{r}}=\hat{y}_{t+1}^{s_{b}} & \text { if } t \leq T-1
\end{array}\right] \forall t \in \mathcal{T}, t \leq \mathbf{t}\left(s_{a}, s_{b}(\mathrm{C} .8)\right.}
\end{aligned}
$$

We complete the proof using Lemma 1. To streamline the use of Lemma 1, we will refer to $s_{a}$ as $s_{0}$. By construction, $\left|\mathcal{D}\left(s_{k}, s_{k+1}\right)\right|=\left|\mathcal{D}\left(s_{k+1}, s_{k}\right)\right|=1$ and $\mathbf{t}\left(s_{k}, s_{k+1}\right)=\mathbf{t}\left(s_{k+1}, s_{k}\right)=T$ for $k \in\{0,1, \ldots, r-1\}$. Thus, from conditions (i) and (iii) of this proposition,

(a) If $s_{k}<s_{k+1}$, then $\left(s_{k}, s_{k+1}\right) \in \mathcal{L}_{T}^{1}$. Hence, vectors $\hat{b}, \hat{x}, \hat{y}, \hat{Z}$ satisfy (13a) for $\left(s, s^{\prime}, t\right)=$ $\left(s_{k}, s_{k+1}, 1\right)$ and (15)-(16) for $\left(s, s^{\prime}\right)=\left(s_{k}, s_{k+1}\right), t \in \mathcal{T}$.

(b) If $s_{k+1}<s_{k}$, then $\left(s_{k+1}, s_{k}\right) \in \mathcal{L}_{T}^{1}$. Hence, vectors $\hat{b}, \hat{x}, \hat{y}, \hat{Z}$ satisfy (13a) for $\left(s, s^{\prime}, t\right)=$ $\left(s_{k+1}, s_{k}, 1\right)$ and $(15)-(16)$ for $\left(s, s^{\prime}\right)=\left(s_{k+1}, s_{k}\right), t \in \mathcal{T}$.

Thus, choosing $\hat{t}=T-1$ for $k \in\{0,1, \ldots, r-1\}$,

(a) If $s_{k}<s_{k+1}$, then sub-vectors $\hat{b}^{s_{k}}, \hat{b}^{s_{k+1}}, \hat{Z}^{s_{k}, s_{k+1}}$ satisfy (9)-(11) for $\left(s, s^{\prime}\right)=\left(s_{k}, s_{k+1}\right)$.

(b) If $s_{k+1}<s_{k}$, then sub-vectors $\hat{b}^{s_{k+1}}, \hat{b}^{s_{k}}, \hat{Z}^{s_{k+1}, s_{k}}$ satisfy (9)-(11) for $\left(s, s^{\prime}\right)=\left(s_{k+1}, s_{k}\right)$.

Thus, using Lemma 1 for $\hat{t}=T-1$ together with

$$
\mathcal{D}\left(s_{a}, s_{1}\right)=\{I-r+1\}, \mathcal{D}\left(s_{1}, s_{2}\right)=\{I-r+2\}, \mathcal{D}\left(s_{2}, s_{3}\right)=\{I-r+3\}, \ldots, \mathcal{D}\left(s_{r-1}, s_{r}\right)=\{I\},
$$

and $s_{0} \equiv s_{a}$, we get

$$
\begin{aligned}
& \bigwedge_{\tau=1}^{t}\left[\left(\neg \hat{b}_{I-r+1, \tau}^{s_{a}}\right) \wedge\left(\neg \hat{b}_{I-r+2, \tau}^{s_{1}}\right) \wedge\left(\neg \hat{b}_{I-r+3, \tau}^{s_{2}}\right) \wedge \cdots \wedge\left(\neg \hat{b}_{I, \tau}^{s_{r-1}}\right)\right] \Leftrightarrow \\
& \bigwedge_{\tau=1}^{t}\left[\left(\neg \hat{b}_{I-r+1, \tau}^{s_{a}}\right) \wedge\left(\neg \hat{b}_{I-r+2, \tau}^{s_{a}}\right) \wedge\left(\neg \hat{b}_{I-r+3, \tau}^{s_{a}}\right) \wedge \cdots \wedge\left(\neg \hat{b}_{I, \tau}^{s_{a}}\right)\right] \quad \forall t \in \mathcal{T}
\end{aligned}
$$

Combining (C.8) and (C.9) together with the fact that $\mathcal{D}\left(s_{a}, s_{b}\right)=\{I-r+1, I-r+2, \ldots, I\}$, we get

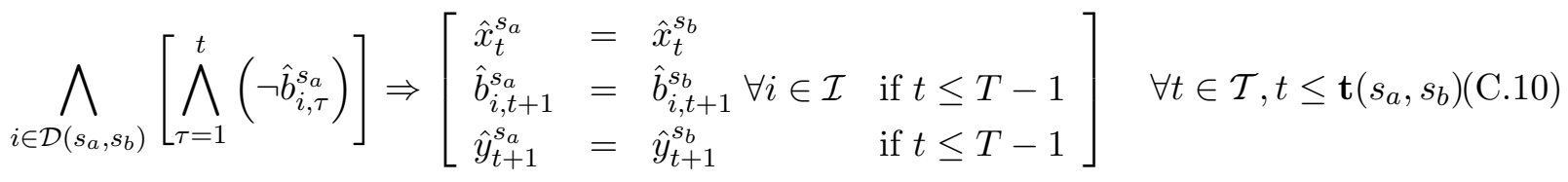

which is simply the re-statement of (15)-(16) for scenario pair $\left(s_{a}, s_{b}\right)$. 
Case 2: $\boldsymbol{t}\left(s_{a}, s_{b}\right)=T$. The line of reasoning used above can be used in this case also. However, by construction above, $\mathcal{D}\left(s_{r}, s_{b}\right)=\emptyset$ and $\mathbf{t}\left(s_{a}, s_{r}\right)=T$. Since $\mathbf{t}\left(s_{a}, s_{b}\right)=T$ in this case, therefore we will have $\mathbf{t}\left(s_{r}, s_{b}\right)=T$ and $\mathcal{D}\left(s_{r}, s_{b}\right)=\emptyset$. Thus, scenarios $s_{r}, s_{b}$ are identical; i.e.,

$$
\left(\xi_{1}^{s_{r}}, \xi_{2}^{s_{r}}, \ldots, \xi_{T}^{s_{r}}, \theta_{1}^{s_{r}}, \theta_{2}^{s_{r}}, \ldots, \theta_{I}^{s_{r}}\right) \equiv\left(\xi_{1}^{s_{b}}, \xi_{2}^{s_{b}}, \ldots, \xi_{T}^{s_{b}}, \theta_{1}^{s_{b}}, \theta_{2}^{s_{b}}, \ldots, \theta_{I}^{s_{b}}\right) .
$$

Thus, we can replace $s_{r}$ by $s_{b}$ in the above proof and use (C.4), (C.5) and (C.6) to obtain (C.8) (without using (C.7)). As in the previous case, Lemma 1 can then be used to obtain (C.10).

Since all we assumed about $s_{a}, s_{b}$ was that $\left|\mathcal{D}\left(s_{a}, s_{b}\right)\right| \geq 1$, we can say that vectors $\hat{b}, \hat{x}, \hat{y}, \hat{Z}$ satisfy (15)-(16) for all $\left(s, s^{\prime}, t\right)$ such that $\left(s, s^{\prime}\right) \in \mathcal{L}^{1+}, t \in \mathcal{T}, t \leq \mathbf{t}\left(s, s^{\prime}\right)$.

\section{Sizes problem: Objective function and constraints represented by $(3)$}

In the sizes problem, $\mathcal{I}$ represents the set of sizes to be produced. We assume that this set is ordered such that if $i, i^{\prime} \in \mathcal{I}$ and $i>i^{\prime}$ then the size corresponding to index $i$ is larger than the size corresponding to index $i^{\prime}$. Hence, delivery of size $i$ can satisfy demand for size $i^{\prime}$. Variable $b_{i, t}^{s}$ represents whether size $i$ is produced in time period $t$ of scenario $s$. Variable $y_{i, t}^{s}$ represents the number of units of size $i$ produced in time period $t$ while $x_{i, i^{\prime}, t}^{s}$ represents the number of units of size $i$ used to satisfy demand of size $i^{\prime}$, where $i \geq i^{\prime} . w_{i, t}^{s}$ represents the inventory of size $i$ at the end of time period $t$.

In the case of the sizes problem, parameters ${ }^{w} c_{t}^{s},{ }^{b} c_{i, t}^{s},{ }^{x} c_{t}^{s}$ and ${ }^{y} c_{t}^{s}$ represent variable inventory costs, fixed production costs, variable substitution costs and variable production costs, respectively. Constraint (3) is represented by (D.1)-(D.7). (D.1) and (D.2) represent variable lower and upper bound constraints respectively, on $y_{i, t}^{s}$. (D.3) represents the constraint that production in any time period should not exceed the total production capacity, represented by $\alpha$. Constraint (D.4) represents the constraint that demands (represented by $\xi_{i^{\prime}, t}^{s}$ ) of all sizes should be satisfied in all time periods. (D.5) computes the inventory for each size at the end of a time period while (D.6) restricts the total inventory at the end of any time period to be not greater than the inventory capacity, represented by $\beta$.

$$
\begin{array}{rlrl}
y_{i, t}^{s} \geq{ }^{y} L_{i} \cdot b_{i, t}^{s} & \forall s \in \mathcal{S}, t \in \mathcal{T}, i \in \mathcal{I} \\
y_{i, t}^{s} \leq{ }^{y} U_{i, t}^{s} \cdot b_{i, t}^{s} & \forall s \in \mathcal{S}, t \in \mathcal{T}, i \in \mathcal{I} \\
\sum_{i \in \mathcal{I}} y_{i, t}^{s} \leq \alpha & & \forall s \in \mathcal{S}, t \in \mathcal{T} \\
\sum_{i \in \mathcal{I}, i \geq i^{\prime}} x_{i, i^{\prime}, t}^{s} \geq \xi_{i^{\prime}, t}^{s} & \forall s \in \mathcal{S}, t \in \mathcal{T}, i^{\prime} \in \mathcal{I}
\end{array}
$$




$$
\begin{array}{rlrl}
\sum_{\substack{\tau \in \mathcal{T} \\
\tau \leq t}}\left(y_{i, \tau}^{s}-\sum_{i^{\prime} \in \mathcal{I}, i \geq i^{\prime}} x_{i, i^{\prime}, \tau}^{s}\right) & =w_{i, t}^{s} & & \forall s \in \mathcal{S}, t \in \mathcal{T}, i \in \mathcal{I} \\
\sum_{i \in \mathcal{I}} w_{i, t}^{s} \leq \beta & & \forall s \in \mathcal{S}, t \in \mathcal{T} \\
w_{i, t}^{s} \geq 0 & \forall s \in \mathcal{S}, t \in \mathcal{T}, i \in \mathcal{I}
\end{array}
$$

Note that the LP relaxation of the model can be tightened by adding constraints (D.8)-(D.10) which exploit a Krarup and Bilde (1977) type dis-aggregation of variables $x_{i, i^{\prime}, t}^{s}$ into variables $x 2_{i, i^{\prime}, \tau, t}^{s}$ for $\tau \leq t$. Physically, variable $x 2_{i, i^{\prime}, \tau, t}^{s}$ represents the number of items of size $i$ produced in time period $\tau$ used to satisfy demand of size $i^{\prime}(\leq i)$ in time period $t(\geq \tau)$. (D.8) relates variables $x_{i, i^{\prime}, t}^{s}$ to dis-aggregated variables $x 2_{i, i^{\prime}, \tau, t}^{s}$, while (D.9) represents variable upper bound constraints on the dis-aggregated variables. (D.10) represents the condition that consumption of any size cannot exceed the production.

$$
\begin{array}{rlrl}
x_{i, i^{\prime}, t}^{s} & =\sum_{\tau \in \mathcal{T}, \tau \leq t} x 2_{i, i^{\prime}, \tau, t}^{s} \forall s \in \mathcal{S}, t \in \mathcal{T} ; i, i^{\prime} \in \mathcal{I}, i \geq i^{\prime} \\
x 2_{i, i^{\prime}, \tau, t}^{s} \leq & { }^{x 2} U_{i, i^{\prime}, \tau, t}^{s} \cdot b_{i, \tau}^{s} & \forall s \in \mathcal{S} ; \tau, t \in \mathcal{T}, \tau \leq t ; i, i^{\prime} \in \mathcal{I}, i \geq i^{\prime} \\
\sum_{\substack{t \in \mathcal{T}, i^{\prime} \in \mathcal{I}, t \geq \tau}} x 2_{i \geq i^{\prime}, \tau, t}^{s} & \leq y_{i, \tau}^{s} & \forall s \in \mathcal{S}, \tau \in \mathcal{T}, i \in \mathcal{I}
\end{array}
$$

Results presented in section 9 are obtained by using constraints (D.1)-(D.10). Note that Jonsbraten et al. (1998) do not consider the variable dis-aggregation and the capacity constraint on total inventory (D.6). 